\title{
Specific Effects and Deconvolution in Submicrometre EPMA: Application to Binary Diffusion
}

\author{
Olivier ARNOULD, François HILD \\ LMT-Cachan, ENS Cachan / CNRS-UMR 8535 / Université Paris VI \\ 61 avenue du Président Wilson, F-94235 Cachan Cedex, France
}

\begin{abstract}
Electron probe (X-ray) microanalysis (EPMA) is nowadays a classical and well-established method for qualitative and (semi)quantitative evaluation of the elemental composition of the (near)surface of a sample on the micrometre scale. This technique can be used to determine concentration profiles due to (inter)diffusion in materials at submicrometre resolution if physical and geometrical effects that occur during the measurement process are accounted for. Standard phenomena are usually corrected by commercial softwares for a homogeneous elemental composition in the analyzed area. However, in the case of a diffusion process on a small scale, the composition is no longer homogeneous and the effect of the hemispherical volume of the X-ray emission on the spatial resolution of the concentration profiles, and consequently on the diffusion coefficients, has to be considered. A radial X-ray distribution associated with the classical depth distribution, $\phi(z)$, allows for the definition of a 2D X-ray emission function for medium to heavy materials. This enables one to study the effect of some geometrical parameters on the measured concentration profile and to propose a method of reconstructing the real weight fraction profile from the measured profile of the X-ray intensities by using regularized deconvolution algorithms.
\end{abstract}

Keywords: EPMA, concentration profiles, average effect, geometrical effects, regularized deconvolution.

\section{Introduction: physical effects during measurements of con- centration profiles}

The evaluation of diffusion coefficients may require high spatial resolution. One of the easiest and most reliable techniques is the Electron Probe MicroAnalysis (EPMA). It consists in measuring the relative intensities of the X-ray wavelengths (WDS), or energies (EDS), emitted by a sample impacted by an electron beam. ${ }^{1}$ It allows for the identification and quantification of elemental compositions if a variety of interactions between electrons, X-rays and atoms are accounted for. The main factors are now well-understood and readily corrected by ZAF or $\phi(\rho z)$ methods for a uniform concentration in the area probed by the electrons and X-ray diffusion in the sample. ${ }^{2}$

*to whom correspondence should be addressed. Fax: +33147402240 - Email: arnould@ lmt.ens-cachan.fr. 
For heterogeneous chemical compounds, i.e., when a phase boundary is present within the volume affected by the electrons or the emitted X-rays, specific phenomena occur. They are summarized in Fig. 1 in which a 1D varying concentration between two pure elements, say $\mathrm{A}$ and $\mathrm{B}$ with $\mathrm{Z}_{\mathrm{B}}>\mathrm{Z}_{\mathrm{A}}$, along $Y$ only is considered. The concentration gradient is, for example, described by the weight fraction in $\mathrm{B}, \mathrm{W}_{\mathrm{B}}(Y)$. The B-rich phase is located for $Y \leq 0$ so that $\mathrm{W}_{\mathrm{B}}(Y)$ decreases with $Y$. This is depicted by the gray level in Fig. 1; the darker, the richer in $\mathrm{B}$-atoms. Note that, since binary diffusion is concerned, $\mathrm{W}_{\mathrm{A}}(Y)=1-\mathrm{W}_{\mathrm{B}}(Y)$ if no porosity appears during the diffusion process ${ }^{3}$ on the diffusion scale (e.g., this can be due to the Kirkendall effect that exists, for example, in a $\mathrm{Ni} / \mathrm{Cu}$ diffusion couple). The origin of the diffusion profile, $\mathrm{O}$, corresponds to the position of an ideal interface before diffusion has occurred (becoming an interphase between $\mathrm{A}$ and $\mathrm{B}$ elements during diffusion). The primary electron beam (acceleration voltage $\mathrm{E}_{0}$ ) is focused at point $\mathrm{I}$ on the surface sample, at a distance $Y=d$ from the initial interface.

The first effect depicted in Fig. 1 is due to the electron diffusion in the specimen that produces a characteristic X-ray emission function whose shape is close to a sphere ${ }^{4}$ of average radius $R_{\mathrm{X}}$ for medium to high $\mathrm{Z}$ materials (i.e., atomic number greater than ten). $2 R_{\mathrm{X}}$ accounts for the diameter of interaction that is an upper bound of the spatial resolution of EPMA techniques. 5, 6,7 The measurement corresponds to an average over this volume leading to what will be referred to as average effect. When dealing with diffusion couples, it has to be evaluated when the diffusion length (i.e., the distance over which diffusion has occurred) is of the order of a characteristic length of the spherical emission volume, i.e., when $\sqrt{2 D t} \approx R_{\mathrm{X}}$, where $D$ is the average diffusion coefficient and $t$ the diffusion time. Furthermore, each part of the emission volume in $\mathrm{S}$ (i.e., the dark gray parallelepipedic volume in Fig. 1) emits characteristic $\left(\mathrm{I}_{\mathrm{A}}\right.$ and $\mathrm{I}_{\mathrm{B}}$ for the $\mathrm{A}$ and $\mathrm{B}$-atoms, respectively) and continuous X-rays in all directions and a part of them travel through the sample surface at $\mathrm{N}$ to reach the spectrometer. Along their way to the surface, X-ray radiations are absorbed in an exponential fashion in a varying chemical composition. This phenomenon introduces a specific absorption correction that is controlled by the detection geometry (take-off angle $\theta$ and orientation angle $\delta$ of the normal of the interface with respect to the spectrometer) and the position $d$ of the primary electron beam in the concentration gradient.

Another important effect is the fluorescence that can be due to the X-ray continuum that can excite both A and B-atoms and the characteristic B-radiations that travel in the specimen and can excite $\mathrm{A}$-atoms at any point $\mathrm{M}$ ( since $\mathrm{Z}_{\mathrm{B}}>\mathrm{Z}_{\mathrm{A}}$ ) leading to fluorescent $\mathrm{X}$-ray emission (noted $\mathrm{I}_{\mathrm{A}}^{\mathrm{f}}$ ) that is measured and modifies the analyzed spectra. ${ }^{8}$ The volume concerned by this effect is much larger than that of the primary radiation and can be predominant over a longer range $^{6}$ especially for the characteristic fluorescence. Classical correction methods take this effect into account for a homogeneous volume but not when different phases or multilayered materials are concerned. These specific cases have been studied ${ }^{9,10,11,12}$ when the primary electron beam does not cross an interface. Recent numerical ${ }^{13,14}$ and analytical ${ }^{8}$ studies have $^{2}$ considered this interface crossing to analyze fluorescence across a phase boundary.

The present study focuses on the first discussed phenomenon, i.e., the average and absorption effect in a chemical gradient when using $\mathrm{K} \alpha \mathrm{X}$-ray lines of the spectrum for determining the elemental chemical composition. The fluorescent radiations will not be taken into account by considering only B-radiations. The average and absorption effects and their deconvolution can be assessed with this radiation since binary diffusion is concerned (i.e., $\mathrm{W}_{\mathrm{B}}$ yields $\mathrm{W}_{\mathrm{A}}$ since 
$\mathrm{W}_{\mathrm{A}}=1-\mathrm{W}_{\mathrm{B}}$ ). Note that, since nickel and copper will be used, the characteristic fluorescence is almost negligible since only the copper $\mathrm{K} \beta$ radiations are able to 'produce' nickel fluorescent radiations. This is all the more true when a 'classical' energy dispersive spectrometer (EDS) is used since it does not have the required accuracy to distinguish Ni-fluorescent radiations from those of the continuous background.

The general expression of the measured $k$-ratio for the element $\mathrm{B}$ when the primary electron beam is at a distance $d$ from the A/B initial interface position is defined ${ }^{1,15}$ by the ratio between the measured intensity in the sample $\mathrm{I}_{\mathrm{B}}(d)$ and the measured intensity in a pure Bsample $\mathrm{I}_{(\mathrm{B})}$

$$
k_{\mathrm{B}}(d)=\frac{\mathrm{I}_{\mathrm{B}}(d)}{\mathrm{I}_{(\mathrm{B})}}
$$

with

$$
\mathrm{I}_{(\mathrm{B})}=\Delta_{\mathrm{I}} \int_{z=0}^{\infty} \int_{x, y=-\infty}^{\infty} \mathscr{F}(x, y, z) \exp \left(-\mu_{\mathrm{B}}^{\mathrm{B}} \rho_{\mathrm{B}} \frac{z}{\sin (\theta)}\right) \mathrm{d} x \mathrm{~d} y \mathrm{~d} z,
$$

and the numerator describes the average effect (Fig. 1)

$$
\mathrm{I}_{\mathrm{B}}(d)=\Delta_{\mathrm{I}} \int_{z=0}^{\infty} \int_{x, y=-\infty}^{\infty} \mathrm{W}_{\mathrm{B}}(y+d) \mathscr{F}(x, y, z) \exp \left[-\int_{l=0}^{z / \sin (\theta)}\left(\mu_{\mathrm{B}}^{\mathrm{AB}} \rho_{\mathrm{AB}}\right)(Y(l)) \mathrm{d} l\right] \mathrm{d} x \mathrm{~d} y \mathrm{~d} z,
$$

with

$$
Y(l)=y+d+l \cos (\theta) \cos (\delta),
$$

where $\Delta_{\mathrm{I}}$ stands for the coefficient arising from the detector efficiency or other material and electron beam parameters (such as the fluorescence yield, Coster-Kronig transitions, the electron beam intensity or the acquisition time), $\mu_{\mathrm{B}}^{\mathrm{AB}}(Y)$ is the mass attenuation coefficient of the B-radiation in the mixed material for a weight fraction $\mathrm{W}_{\mathrm{B}}(Y)$ that is obtained by a rule of mixtures 6,15

$$
\mu_{\mathrm{B}}^{\mathrm{AB}}(Y)=\mathrm{W}_{\mathrm{B}}(Y) \mu_{\mathrm{B}}^{\mathrm{B}}+\left(1-\mathrm{W}_{\mathrm{B}}(Y)\right) \mu_{\mathrm{B}}^{\mathrm{A}},
$$

and $\rho_{A B}(Y)$ is the relative density for the material with a weight fraction $\mathrm{W}_{\mathrm{B}}(Y)$ that is obtained by a reverse rule of mixtures

$$
\rho_{\mathrm{AB}}(Y)^{-1}=\mathrm{W}_{\mathrm{B}}(Y) \rho_{\mathrm{B}}^{-1}+\left(1-\mathrm{W}_{\mathrm{B}}(Y)\right) \rho_{\mathrm{A}}^{-1},
$$

$\mathscr{F}(x, y, z)$ is a $3 \mathrm{D}$ characteristic $\mathrm{X}$-ray emission distribution corresponding to the ratio between the emitted intensity of the elemental volume at $\mathrm{S}$ (Fig. 1) to that produced by the same isolated volume. It depends a priori on the local concentration, but this can be neglected if the two elements, $\mathrm{A}$ and $\mathrm{B}$, have close atomic numbers. When a $\mathrm{Ni}(\mathrm{A}) / \mathrm{Cu}(\mathrm{B})$ couple is considered, $\mathscr{F}$ can be assumed to be constant and has to be determined to study the average effect and other phenomena related with the absorption or geometrical defaults. This spatial distribution function $\mathscr{F}$, for a pure material, in the local coordinates $(\mathrm{I}, x, y, z)$ is axisymmetric along the depth coordinate $z$ and, as binary diffusion along $Y$ is concerned, all the functions used in Eq. (3) only depend on $y$ and $z$. Integrating over $x$ involves only $\mathscr{F}$ so that a $2 D$ characteristic emission function $F$ can be defined as

$$
F(y, z)=\int_{x=-\infty}^{\infty} \mathscr{F}(x, y, z) \mathrm{d} x .
$$

Consequently Eqs. (2) and (3) become

$$
\mathrm{I}_{(\mathrm{B})}=\Delta_{\mathrm{I}} \int_{z=0}^{\infty} \int_{y=-\infty}^{\infty} F(y, z) \exp \left(-\mu_{\mathrm{B}}^{\mathrm{B}} \rho_{\mathrm{B}} \frac{z}{\sin (\theta)}\right) \mathrm{d} y \mathrm{~d} z
$$


and

$$
\mathrm{I}_{\mathrm{B}}(d)=\Delta_{\mathrm{I}} \int_{z=0}^{\infty} \int_{y=-\infty}^{\infty} \mathrm{W}_{\mathrm{B}}(y+d) F(y, z) \exp \left[-\int_{l=0}^{z / \sin (\theta)}\left(\mu_{\mathrm{B}}^{\mathrm{AB}} \rho_{\mathrm{AB}}\right)(Y(l)) \mathrm{d} l\right] \mathrm{d} y \mathrm{~d} z .
$$

This 2D characteristic emission function is characterized by its well-known depth part $^{1}$ that must be associated with a lateral emission distribution for the present study.

\section{Lateral X-ray emission distributions}

A Monte Carlo computation ${ }^{16}$ of the shape of the $2 \mathrm{D}$ X-ray emission distribution for pure copper is shown in Fig. 2 together with the lateral and depth distributions. The distribution function along the lateral coordinate $y$ and the depth $z$ is close to a Gaussian for the material considered $^{7}$ that has the following property

$$
F(y, z) \approx \psi(y) \phi^{*}(z)
$$

where $\psi(y)$ is a lateral X-ray distribution that accounts for the radial spread of the electrons and $\phi^{*}(z)$ a depth-distribution function. Moreover, if the distribution $\psi$ is normalized (i.e., the mean number of ionization per electron is only contained in $\phi^{*}(z)$ )

$$
\int_{y=-\infty}^{\infty} \psi(y) \mathrm{d} y=1
$$

then $\phi^{*}$ is equivalent to the well-known depth distribution function $\phi(z)$ that is defined by the ratio between the X-ray intensity emitted by a thin layer of thickness $\mathrm{d} z$ at a depth $z$ (Fig. 2) and that produced in an isolated thin layer ${ }^{1}$

$$
\phi(z)=\int_{y=-\infty}^{\infty} F(y, z) \mathrm{d} y=\int_{y=-\infty}^{\infty} \psi(y) \phi^{*}(z) \mathrm{d} y=\phi^{*}(z) \int_{y=-\infty}^{\infty} \psi(y) \mathrm{d} y=\phi^{*}(z)
$$

when considering Eq. (11). The model used for describing $\phi(z)$ is given by ${ }^{17,18}$

$$
\phi(z)=\underbrace{\gamma \exp \left(-(\alpha \rho z)^{2}\right)}_{\text {Gaussian }} \overbrace{\left[1-\frac{\gamma-\phi(0)}{\gamma} \exp (-\beta \rho z)\right]}^{\text {Transient function }},
$$

where the Gaussian accounts for the random walk along $z$ of the electrons producing X-rays and the transient function describes the change of the electron path from a collimated beam to an entire scattered process at a depth $z_{r}$. This model gives good results in the present case with modified parameters for medium to high atomic number materials. ${ }^{19,20}$ Note that $z_{r}$ corresponds to the position where the transient function in $\phi(z)$ becomes negligible, i.e.,

$$
z_{r}=-\frac{1}{\beta \rho} \ln \left(\xi \frac{\gamma}{\gamma-\phi(0)}\right)
$$

where $\xi$ is the residual percentage of the transient function.

The lateral distribution function can be defined in a similar way as $\phi(z)$, i.e., $\psi(y)$ can be defined as the ratio between the X-ray intensity emitted by a thin layer of thickness $\mathrm{d} y$ 
at $y$ coordinate and that produced in an isolated thin layer (Fig. 2) and normalized following Eq. (11). This distribution function has not received a lot of attention. However, considering the electron diffusion process in the specimen as it is described in the previous paragraph to explain the shape of $\phi(z)^{17}$ and, in particular, the isotropic scattering of the electrons at depth $z_{r}$, leads us to assume that the lateral distribution function for $y=0$ to $\infty$ is close to the depth one for $z=z_{r}$ to $\infty$ (this is illustrated by the identical dashed lines in the $\psi$ and $\phi$ distributions in Fig. 2). This assumption allows us to write $\psi(y)$ as the Gaussian part of the $\phi(z)$ distribution with a coordinate shift equal to $z_{r}$

$$
\psi(y)=\underbrace{\frac{\alpha \rho}{\sqrt{\pi} \operatorname{erfc}\left(\alpha \rho z_{r}\right)}}_{\text {normalizing factor }} \exp \left[-\left(\alpha \rho\left(|y|+z_{r}\right)\right)^{2}\right],
$$

where the pre-exponential parameter is a normalizing factor to satisfy Eq. (11) and the absolute value of $y$ yields the symmetry of the distribution with respect to $y=0$. This distribution can be experimentally obtained from the B-element X-ray intensity measurements when crossing a perfect interface between two close materials (e.g., a $\mathrm{Ni}(\mathrm{A}) / \mathrm{Cu}(\mathrm{B})$ couple) with a spectrometer position $\delta$ such that all the measured B-radiations are absorbed in B (see Section 3.1), i.e., $|\delta| \geq \pi / 2$, and Eq. (3) yields

$$
\mathrm{I}_{\mathrm{B}}(d)=\Delta_{\mathrm{I}} \int_{z=0}^{\infty} \int_{y=-\infty}^{-d} \psi(y) \phi(z) \exp \left(-\mu_{\mathrm{B}}^{\mathrm{B}} \rho_{\mathrm{B}} \frac{z}{\sin (\theta)}\right) \mathrm{d} y \mathrm{~d} z .
$$

If the standard measurement, $\mathrm{I}_{(\mathrm{B})}$, is carried out under the same condition as $\mathrm{I}_{\mathrm{B}}(d)$, considering the $k$-ratio allows one to simplify the numerator and the denominator by the integration over $z$ and the $\Delta_{\mathrm{I}}$ factors, then Eq. (1) becomes

$$
k_{\mathrm{B}}(d)=\frac{\int_{y=-\infty}^{-d} \psi(y) \mathrm{d} y}{\int_{y=-\infty}^{\infty} \psi(y) \mathrm{d} y}=\int_{y=-\infty}^{-d} \psi(y) \mathrm{d} y,
$$

since the denominator is equal to 1 in accordance with Eq. (11). Thus, deriving Eq. (17) with respect to $d$ yields

$$
\psi(d)=-\frac{\partial k_{\mathrm{B}}}{\partial d}(d)
$$

An example of this method, obtained with an electrodeposited $\mathrm{Ni}(\mathrm{A}) / \mathrm{Cu}(\mathrm{B})$ diffusion couple without diffusion, is shown in Fig. 3. Fitting results of Eqs. (15) and (17) on $k$-ratio measurements by EDS across a perfect interface allows for the determination of the unknown parameters of the lateral distribution (i.e., $\alpha \rho$ and $z_{r}$ ) as depicted in Fig. 3(a). The resulting lateral distribution is checked in Fig. 3(b) by plotting the latter and the derivative of the EDS measurement following Eq. (18). Curve fitting of the EDS measurements shown in this figure validates the choice of the lateral distribution since the value of $\alpha \rho$ found for $\psi(y)$ is very close to that obtained with the closed-form formulas ${ }^{19,20}$ for $\phi(z)$. Furthermore, a value of $\xi$ of the order of $0.5 \%$ seems to lead to a consistent value of $z_{r}$ (Eq. (14)) when compared to the $2 \mathrm{D} \mathrm{X}$-ray distribution obtained by Monte Carlo computations (Fig. 2). The lateral spread expressed by Eq. (15) is in good agreement with other numerical computations ${ }^{16}$ and with the shape obtained at least for other medium to heavy materials ${ }^{21,22}$ when considering the K-lines.

A simplified expression is generally used for the lateral spread by replacing $\psi(y)$ by a Gaussian distribution ${ }^{23,24}$

$$
g_{\sigma}(y)=\frac{1}{\sqrt{2 \pi} \sigma} \exp \left(-\frac{y^{2}}{2 \sigma^{2}}\right)
$$


where $\sigma$ is the standard deviation of this distribution. Moreover, the corresponding $k_{\mathrm{B}}$-ratio has a closed-form expression, by using Eq. (17)

$$
k_{\mathrm{B}}(d)=\int_{y=-\infty}^{-d} g_{\sigma}(y) \mathrm{d} y=\frac{1}{2}\left[1-\operatorname{erf}\left(\frac{d}{\sqrt{2} \sigma}\right)\right] .
$$

By simply fitting the measurement points with this last expression as it has been performed in Fig. 3(a), one can tune $\sigma$ (i.e., $\sigma=0.27 \mu \mathrm{m}$ for nickel or copper materials with $\mathrm{E}_{0}=25 \mathrm{keV}$ ). The lateral distribution $g_{\sigma}(y)$ corresponding to this fit is shown in Fig. 3(b) and can be compared with $\psi(y)$. A negligible difference is obtained between $g_{\sigma}(y)$ and $\psi(y)$ for the $k_{\mathrm{B}}$-ratio (Fig. 3(a)), which is less accurate for the distribution function (Fig. 3(b)) but it still remains acceptable.

Finally, it is important to note that the size of the beam diameter may have to be taken into account contrary to what happens for the depth behavior. This is all the more important when WDS measurements are carried out where the probe diameter can be of the same order of magnitude as the interaction volume. However, for EDS measurements of medium to heavy materials in an SEM, since the probe current is generally low (i.e., between about 0.1 to $1 \mathrm{nA}$ ) and the acceleration voltage (reasonably) high (to excite the K-shell of the considered species), the effect of the electron beam diameter on the lateral spread of the electron remains negligible ${ }^{25}$ and will be neglected in the lateral X-ray distribution used later on. Let us now focus on some 'geometrical default' effects on the measurements that will be neglected in the last part where a general algorithm is proposed for the deconvolution of the average effect.

\section{Geometrical effects on EPMA measurements}

\subsection{Position of the spectrometer with respect to the diffusion axis}

The position of the spectrometer with respect to the diffusion axis plays an important role in the measured $k$-ratio. This arises from the specific absorption term in Eq. (3). It is interesting to study the change of the $k_{\mathrm{B}}$-ratio with $\delta$ for a perfect boundary between $\mathrm{A}$ and $\mathrm{B}$, i.e., when no diffusion has occurred. Equation (3) reduces to

$$
\mathrm{I}_{\mathrm{B}}(d)=\Delta_{\mathrm{I}} \int_{z=0}^{\infty} \int_{y=-\infty}^{-d} \psi(y) \phi(z) \exp [-\mathscr{A}(z, \delta)] \mathrm{d} z \mathrm{~d} y
$$

with

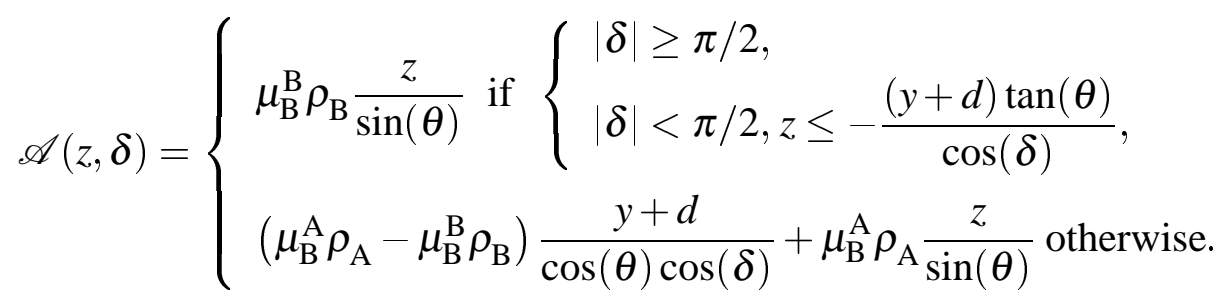

The effect of the spectrometer position on the global apparent concentration curve (i.e., the $k_{\mathrm{B}}$-ratio) is depicted in Fig. 4(a) where the variation of the $k_{\mathrm{B}}$-ratio is plotted vs. the position $d$ of the measurement point from the interface between $\mathrm{A}$ and $\mathrm{B}$ for different mass absorption coefficients and spectrometer angular positions $\delta$. A strong dependence of the (simulated) measurements with $\delta$ is observed even for low absorption coefficients like those of a $\mathrm{Ni}(\mathrm{A}) / \mathrm{Cu}(\mathrm{B})$ couple. This introduces an asymmetry in the apparent concentration profile that could lead to 
wrong interpretations of the diffusion coefficient (see Section 3.2.2). This effect is related with the mass absorption coefficient $\mu_{\mathrm{B}}^{\mathrm{A}}$ (when dealing with B-radiations). The combined effect of $\delta$ and this coefficient is depicted in Fig. 4(b) where the change of the measured $k_{\mathrm{B}}$-ratio for $d=0$ with $\delta$ and $\mu_{\mathrm{B}}^{\mathrm{A}}$ is plotted. This result shows a strong dependence of the measurement with these two parameters except when $|\delta|$ is greater than or equal to $\pi / 2$, i.e., when the spectrometer is on the same side as B and no absorption occurs in A. This observation leads us to conclude that, if $\mathrm{A}$ and $\mathrm{B}$-radiations are both measured, then the best spectrometer position is $\delta=\pi / 2$, i.e., the spectrometer must be parallel to the initial interface between A and B (and it must be close to the center of this interface). Consequently, in all the following developments, it is assumed that $\delta=\pi / 2$.

\subsection{Non-perpendicular phase boundary}

\subsubsection{A simplified X-ray emission distribution}

In all the previous (and following) developments, the initial interface is implicitly assumed to be perfectly perpendicular to the sample surface. The effect of its non-perpendicularity on concentration profiles has to be evaluated. It is possible to estimate the sensitivity of the measurement on the real interface orientation by simplifying the characteristic distribution function of the X-rays. As mentioned in Section 2, for the materials considered, the electron paths tend to an entire isotropic scattered process at a depth $z_{r}$. This allows us to approximate the 3D $\mathrm{X}$-ray emission distribution, in this section, by a sphere of radius $R_{\mathrm{S}}$ centered at a depth $z_{r}$ from the sample surface as depicted in Fig. 5(a). The discussion is limited here to the case of a perfect phase boundary without diffusion. In the following, the absorption, the atomic number (i.e., dependence of the $\mathrm{X}$-ray emission distribution with $\mathrm{Z}$ and therefore with $\mathrm{W}_{\mathrm{B}}$ ) and the fluorescence effects are neglected. The $k_{\mathrm{B}}$-ratio for a perpendicular interface is then given by the ratio between the volume of the sphere that is in the B-phase to the entire partial-spherical volume

$$
k_{\mathrm{B}}(d)=\left\{\begin{array}{llc}
1 & \text { when } \quad d \leq-R_{\mathrm{S}}, \\
1-2 \frac{v_{0}(d)}{\mathrm{V}_{\mathrm{tot}}} & \text { when }-R_{\mathrm{S}}<d \leq-d^{*}, \\
1-\frac{1}{\mathrm{~V}_{\mathrm{tot}}}\left[v_{z_{r}}(d)+v_{0}(d)\right] & \text { when }-d^{*}<d \leq d^{*}, \\
2 \frac{v_{0}(-d)}{\mathrm{V}_{\mathrm{tot}}} & \text { when } & d^{*}<d \leq R_{\mathrm{S}}, \\
0 & \text { when } & R_{\mathrm{S}}<d,
\end{array}\right.
$$

where $d^{*}=\sqrt{R_{\mathrm{s}}^{2}-z_{r}^{2}}, \mathrm{~V}_{\text {tot }}=\pi\left(2 R_{\mathrm{S}}-z_{r}\right)\left(R_{\mathrm{S}}+z_{r}\right)^{2} / 3$ stands for the entire volume of the part of the sphere that is under the surface, and

$$
v_{z_{r}}(d)=\int_{0}^{z_{r}}\left[\left(R_{\mathrm{s}}^{2}-z^{2}\right) \cos ^{-1}\left(-\frac{d}{\sqrt{R_{\mathrm{S}}^{2}-z^{2}}}\right)+d \sqrt{R_{\mathrm{s}}^{2}-z^{2}-d^{2}}\right] \mathrm{d} z,
$$

is the volume of the sphere in the A-phase that lies between $z=0$ and $z=z_{r}$ (the dark grey surface in Fig. 5(a)) whereas

$$
v_{0}(d)=\frac{\pi}{3}\left(d+R_{\mathrm{S}}\right)^{2}\left(R_{\mathrm{S}}-\frac{d}{2}\right)
$$


describes the part of the hemisphere (i.e., for $z>z_{r}$ ) that lies in the A-phase (the light grey surface in Fig. 5(a)). This simplified description of the X-ray emission distribution is still difficult to handle analytically when dealing with a non-perpendicular interface. Furthermore, the effect of a non-zero depth shift $z_{r}$ is negligible as shown in Fig. 5(b) where the root mean square error between a hemispherical distribution $\left(z_{r}=0\right.$ in Eq. (23)) and a partial-spherical one $\left(0<z_{r} \leq R_{\mathrm{S}}\right.$ in Eq. (23)) is plotted vs. the dimensionless depth shift $z_{r} / R_{\mathrm{S}}$ for a perfectly perpendicular interface. The maximum error is found to be about $0.18 \%$, which is negligible. Note that it is equivalent to consider a hemisphere (i.e., $z_{r}=0$ ) or a sphere (i.e., $z_{r}=r$ ) for a perpendicular interface. However, even if it is simpler to consider the case of the sphere for studying the effect of a non-perpendicular interface, it always yields symmetric apparent concentration profiles for any interface orientation. This is in contradiction with the asymmetric effect observed experimentally for a non-perpendicular interface as it will be shown in the next part. Consequently, the hemispherical distribution is chosen and is represented by the case $z_{r}=0$ so that Eq. (23) reduces to ${ }^{26}$

$$
k_{\mathrm{B}}(d)=\left\{\begin{array}{lll}
1 & \text { when } \quad d<-R_{\mathrm{s}}, \\
1-\frac{3 v_{0}(d)}{2 \pi R_{\mathrm{S}}^{3}} & \text { when } & |d| \leq R_{\mathrm{s}}, \\
0 & \text { when } \quad R_{\mathrm{S}}<d .
\end{array}\right.
$$

Note that the radius of the hemisphere, $R_{\mathrm{s}}$, can be obtained by fitting the $k$-ratio predicted by Eq. (26) on the measured $k$-ratio when crossing a perfect interface similarly to what has been performed in Section 2 with more realistic X-ray distributions and accounting for absorption. An example is given in Fig. 6 where EDS measurements of the $\mathrm{Cu} k$-ratio when crossing a perfect interface in a Ni/Cu couple with a primary electron beam acceleration voltage $\mathrm{E}_{0}=25 \mathrm{keV}$ leads to $R_{\mathrm{S}}=0.56 \mu \mathrm{m}$ that is close to the effective spatial resolution (see Section 4.3). This value is less than the radius $R_{\mathrm{X}}$ (close to $1 \mu \mathrm{m}$ in the present case, see Fig. 2), since the latter accounts for the whole area affected by the X-ray emission whereas $R_{\mathrm{S}}$ accounts for an equivalent constant $\mathrm{X}$-ray distribution. It is interesting to note that the measurements and the $k$-ratio predicted by the hemisphere distribution are close in spite of the simplicity of this distribution and the assumptions made.

\subsubsection{Asymmetric effect}

Let us now consider an interface oriented by an angle $\zeta$ (assumed to be positive) with respect to the normal of the sample surface (Fig. 7(a)), the $k_{\mathrm{B}}$-ratio is expressed as

$$
k_{\mathrm{B}}(d)= \begin{cases}1 & \text { when } d<-R_{\mathrm{s}} / \cos (\zeta), \\ 1-2 \frac{v_{0}(d \cos (\zeta))}{\mathrm{V}_{\mathrm{tot}}} & \text { when }-R_{\mathrm{S}} / \cos (\zeta) \leq d<-R_{\mathrm{s}}, \\ 1-\frac{1}{\mathrm{~V}_{\mathrm{tot}}}\left[v_{0}(d \cos (\zeta))+v_{\zeta}(d)\right] & \text { when }-R_{\mathrm{s}} \leq d<R_{\mathrm{s}}, \\ 0 & \text { when } \quad R_{\mathrm{S}}<d,\end{cases}
$$

where $\mathrm{V}_{\text {tot }}=2 \pi R_{\mathrm{s}}^{3} / 3$ stands for the complete volume of the hemisphere,

$$
v_{\zeta}(d)=\frac{2 R_{\mathrm{s}}^{3}}{3} \int_{\varphi=0}^{\zeta}\left(1-\left(\frac{d \cos (\zeta)}{r \cos (\varphi)}\right)^{2}\right)^{3 / 2} \mathrm{~d} \varphi
$$


is the volume of the hemisphere that corresponds to the error introduced by the non-perpendicularity (represented by the dark grey volume in Fig. 7(a)), and $v_{0}$ is the same part of the hemisphere as described in the previous part (see Eq. (25) and the light grey surface in Fig. 7(a)).

An example of the measured $k$-ratio predicted by Eq. (27) is given in Fig. 7(b) for two misorientation angles compared with a perpendicular interface. The change of the profile with $\zeta$, i.e., its asymmetry, is similar to what has been depicted for the effect of the spectrometer position $\delta$ in Section 3.1. An interesting parameter for studying these apparent concentration profiles is the skewness, $\gamma_{1}$, which accounts for the dissymmetry of $k_{\mathrm{B}}(d)$ that is the consequence of a non-perpendicular interface. It can be obtained from the derivative of $k_{\mathrm{B}}(d)$ with respect to $d$

$$
\gamma_{1}=\int_{-R_{\mathrm{s}} / \cos (\zeta)}^{R_{\mathrm{S}}} \frac{(d-\bar{d})^{3}}{\mathscr{V}^{3 / 2}} \frac{\partial k_{\mathrm{B}}(d)}{\partial d} \mathrm{~d} d
$$

where $\bar{d}$ and $\mathscr{V}$ stand for the mean and the variance of the distribution $\partial k_{\mathrm{B}}(d) / \partial d$, respectively. The change of the coefficient of asymmetry (i.e., the skewness) with the default of perpendicularity, $\zeta$, is shown in Fig. 8(a) where a corrected skewness, $\gamma_{1}-0.42 \zeta$, is plotted versus the misorientation angle $\zeta$. This plot shows that it is difficult to detect the asymmetry when $\zeta$ is less than or equal to $\pi / 10$. This corresponds to an absolute skewness of about 0.13 . A validation of the use of the skewness to detect a non-perpendicular interface has been obtained by measuring the apparent concentration profile of copper in an electrodeposited multilayered $\mathrm{Ni} / \mathrm{Cu}$ tube with perfectly planar interfaces (see the schematic in Fig. 8(a)). Cutting this tube with different radial shifts from the axis allows one to obtain several interface misorientations. Furthermore, these interfaces remain perfectly planar on the micrometre scale. The (local) misorientation angle is estimated by using the low depth of field for high magnification of an optical microscope (i.e., about $0.3 \mu \mathrm{m}$ for a dry objective with a numerical aperture of 0.9 and magnification of 100) and the micrometre-calibrated focusing mechanism for measuring the vertical displacement of the sample ( $2 \mu \mathrm{m}$ graduations). The misorientation angle is measured inside the tube and not at the interface but this introduces a negligible error as the ratio between the thickness and the average radius of the tube is low in the present case (about $10^{-2}$ ). In the following example, it introduces an error of about $1^{\circ}$ that is less than the error due to the value of the depth of field and the precision of the focusing stage (about $5^{\circ}$ ). Furthermore, the local misorientation can be checked by measuring the ratio between the apparent radius of the interface and the real radius. Finally, the measurement with the EDS spectrometer leads to an average misorientation angle (since the real interface is a cylinder) on a depth of about $1 \mu \mathrm{m}$ that induces a negligible difference between this average angle and the tangential angle at the surface.

Figure 8(b) shows the resulting $k_{\mathrm{B}}$-ratio obtained on an extreme case such that the optically estimated misorientation angle is equal to $0.4 \pi^{ \pm 0.1}$ (i.e., $\zeta \approx 70^{ \pm 5^{\circ}}$ ). The mean derivative over 3 consecutive EDS measurement points is first determined and the skewness is derived using Eq. (29) $\left(\gamma_{1}=0.244\right.$ for the example in Fig. 8(b)). This leads in Fig. 8(a) to a misorientation angle of about $0.44 \pi$ (i.e., $\zeta \approx 80^{\circ}$ ) that is confirmed by comparing the corresponding curve using Eq. (27) and the measurements in Fig. 8(b). A difference of $14 \%$ is found between 'optical' and EDS measurements. This result is satisfactory when considering the simplification in the X-ray emission distribution, the high misorientation angle and when considering the fact that the interface may not be really 'perfect'.

It is interesting to analyze what kind of interdiffusion coefficient the asymmetric effect induces if no correction is performed, that is when the measured $k$-ratio is taken to be the weight 
fraction W (this analysis can be applied to account for the effect of the spectrometer position in Section 3.1). The results are shown in Fig. 9 where the ratio between the apparent interdiffusion coefficient for a misorientation angle $\zeta$, say $D_{\zeta}^{*}$, and that for a perfect interface, say $D_{0}^{*}$ is plotted with respect to the apparent concentration $\mathrm{W}_{\mathrm{B}}^{*}=k_{\mathrm{B}}$. The apparent interdiffusion coefficient has been obtained by the Boltzmann-Matano method ${ }^{3}$ applied to the $k$-ratio predicted by Eq. (27). It is important to remember that this method is not very accurate for extreme concentrations (i.e., when $\mathrm{W}_{\mathrm{B}} \rightarrow 0$ or $\mathrm{W}_{\mathrm{B}} \rightarrow 1$ that corresponds here to $k_{\mathrm{B}} \rightarrow 0$ or $k_{\mathrm{B}} \rightarrow 1$ ) and that in these cases, the Grube method should be used. ${ }^{3}$ The asymmetry of the apparent concentration due to the misoriented interface leads to a concentration-dependent interdiffusion coefficient similar to what is generally obtained for a $\mathrm{Ni}(\mathrm{A}) / \mathrm{Cu}(\mathrm{B})$ couple $^{27}$ when $\zeta$ is negative (i.e., $D$ increases with $\mathrm{W}_{\mathrm{B}}$ ). The opposite trend occurs when $\zeta$ is positive. These trends can be reverted when $|\zeta|$ becomes greater than about $0.3 \pi$. This phenomenon is linked with a change in asymmetry of the concentration profile with respect to the misorientation angle $\zeta$ (Fig. 8(a)). The asymmetric effects due to the detector angle position $\delta$ and the interface misorientation $\zeta$ are not very obvious to handle accurately even though no diffusion has occurred. This would be all the more difficult when diffusion is studied. Consequently, $\delta$ should be close to $\pi / 2$ and $\zeta$ to 0 . These assumptions are made in the following analysis and the geometrical configuration of the sample checked before any measurements.

\section{General deconvolution of concentration profiles}

\subsection{Introduction and simplified result}

The aim of the present EPMA measurements is to obtain the interdiffusion coefficient(s) for the considered species. The subject of this part is to obtain the real diffusion coefficient from the real weight fraction curve $\mathrm{W}_{X}(Y)$ (where $X$ stands for material A or $\mathrm{B}$ ). This weight fraction must be extracted, by an adapted deconvolution technique, from the measurement $m_{X}(d)$ of the real $k$-ratio (see Eq. (1)) for the element $X$ at point $d$, say $\bar{k}_{X}\left(\mathrm{~W}_{X}(Y), d\right)$, with

$$
m_{X}(d)=\bar{k}_{X}\left(\mathrm{~W}_{X}(Y), d\right)+n(d),
$$

where $n(d)$ is the measurement noise that contains the error arising from the detector or some surface sample roughness as well as that from the instability of the primary electron beam current for example. In this section, the analyzed sample is assumed to be well prepared and positioned with respect to the spectrometer, i.e., $\delta$ is close to $\pi / 2$ and $\zeta$ to 0 (see Sections 3.1 and 3.2.2, respectively).

The physical effect that mainly affects the measured curve in the present case is the so-called 'average' effect. It can be qualitatively assessed by neglecting the absorption effect and the fluorescent radiations for a nearly concentration-independent diffusion coefficient. Then, using the approximate lateral X-ray Gaussian-like distribution $g_{\sigma}$ (Eq. (19)) allows one to show $28,29,26$ (Appendix A) that the apparent interdiffusion coefficient obtained with the raw measurements curve, $D^{*}$, is related to the real one, $D$, by

$$
D^{*}=D+\frac{\sigma^{2}}{2 t}
$$

where $\sigma$ is the standard deviation of the lateral X-ray distribution and $t$ the diffusion time. Equation (31) illustrates the average effect on the measured interdiffusion coefficient that leads 
to an overestimation of the real diffusion coefficient and particularly when $D \leq \sigma^{2} / 2 t$. This equation could be used to obtain real diffusion coefficients but they are generally concentrationdependent. ${ }^{3}$ Furthermore, the absorption effect and the fluorescent radiations cannot be neglected in general. Thus, a more complex deconvolution technique must be used.

\subsection{Deconvolution procedures}

The real measurements yield discrete values of Eq. (30) in $N$ displacement points $d_{i}$

$$
\mathrm{m}_{X}^{i}=\overline{\mathrm{k}}_{X}^{i}+\mathrm{n}_{i}
$$

where $\mathrm{m}_{X}^{i}$ is the EPMA measurement at point $d_{i}$ that is set in the vector $\underline{\mathrm{m}}_{X}, \overline{\mathrm{k}}_{X}^{i}$ the real $k$ ratio at point $d_{i}$ and $\mathrm{n}_{i}$ the noise at point $d_{i}$. The noise is assumed to be Gaussian of mean value 0 (i.e., no systematic error during measurements) with the same standard deviation $\sigma_{\mathrm{n}}$ for each point. An estimated value of about 0.01 is found in all the present measurements. It is important to note that this assumption can have very important consequences on the quality of the deconvolution, since it is very sensitive to $\mathrm{n}_{i},{ }^{30,31}$ and that it can be improved by determining the true distribution of noise at each measurement point if sufficient measurements are carried out or by including this distribution as unknown parameters in the deconvolution procedure. ${ }^{30,31}$

The unknown weight fraction $\mathrm{W}_{X}(Y)$ is assumed to be stepwise constant between two measurement points (see Fig. 13 in Appendix B) and values at the measurement points $d_{i}$, say $\mathrm{W}_{X}^{i}$, are set in a vector $\mathrm{W}_{X}$. This assumption leads us to write the calculated $k$-ratio at point $d_{i}$ for the element $X$, say $\mathrm{k}_{X}^{i}$, from the unknown weight fraction as

$$
\mathrm{k}_{X}^{i}=\sum_{j=1}^{N} \mathrm{H}_{X}^{i j}\left(\underline{\mathrm{W}}_{X}\right) \mathrm{W}_{X}^{j}
$$

where $\underline{\underline{H}}_{X}$ stands for the transfer matrix for the measurements of $X$-ray radiations and consists of discrete values of the absorption, average and fluorescent effects. Note that this matrix is not necessarily square, i.e., the number of points for the discretization of the weight fraction curve could be different from (i.e., greater than) the number of measurements. This may be considered as impossible since the number of unknowns is greater than the number of data. Yet it is possible if the functional used for the deconvolution includes additional information on the solution.

The basic idea of deconvolution is to minimize the usual mean square error $\varepsilon$ between the calculated and measured data

$$
\varepsilon^{2}=\sum_{X=\mathrm{A}, \mathrm{B}}\left(\underline{\mathrm{m}}_{X}-\underline{\mathrm{k}}_{X}\right)^{t} \cdot \underline{\underline{\mathrm{C}}}^{-1} \cdot\left(\underline{\mathrm{m}}_{X}-\underline{\mathrm{k}}_{X}\right)=\sum_{i=1}^{N} \sum_{X=\mathrm{A}, \mathrm{B}} \frac{\left(\mathrm{m}_{X}^{i}-\mathrm{k}_{X}^{i}\right)^{2}}{\sigma_{\mathrm{n}}^{2}},
$$

where $\underline{\underline{C}}$ is the covariant matrix of the noise distribution that is assumed to be equal to $\sigma_{\mathrm{n}}^{2} \underline{\underline{l}}$, where $\underline{\underline{I}}$ is the identity matrix in the present case, and $\cdot^{t}$ the matrix transposition. Only Aradiations are affected by the fluorescent ones and this leads to time-consuming computations and a transfer matrix $\underline{\underline{H}}_{A}$ that is not very easy to determine. Therefore, the deconvolution will generally only take the $\mathrm{B}$-radiations into account and the previous equation reduces to

$$
\varepsilon^{2}\left(\underline{\mathrm{m}}_{\mathrm{B}}, \underline{\mathrm{W}}_{\mathrm{B}}\right)=\sum_{i=1}^{N} \frac{\left(\mathrm{m}_{\mathrm{B}}^{i}-\mathrm{k}_{\mathrm{B}}^{i}\right)^{2}}{\sigma_{\mathrm{n}}^{2}}
$$


where the transfert matrix for the B-radiations, $\underline{\underline{H}}_{B}$ given in Appendix $\mathrm{B}$, is used to compute $\underline{k}_{B}$ with Eq. (33). The computation of the A-radiations ${ }^{8}$ can be used to check the quality of the deconvolution. Note that the deconvolution is more accurate if the A-radiations are considered too as they give extra information without any additional unknowns since $\mathrm{W}_{\mathrm{A}}=1-\mathrm{W}_{\mathrm{B}}$ and $k_{\mathrm{A}} \neq 1-k_{\mathrm{B}}$.

This way of reconstructing the real weight fraction profile (i.e., $\underline{\mathrm{W}}_{\mathrm{B}}$ from $\underline{\mathrm{m}}_{\mathrm{B}}$ by minimizing Eq. (35)) leads, in the general case of a non-square transfer matrix, to the approximate solution

$$
\underline{\hat{\mathrm{W}}}_{\mathrm{B}}=\left(\underline{\underline{\mathrm{H}}}_{\mathrm{B}}^{t} \cdot \underline{\underline{\mathrm{H}}}_{\mathrm{B}}\right)^{-1} \cdot \underline{\underline{\mathrm{H}}}_{\mathrm{B}}^{t} \cdot \underline{\mathrm{m}}_{\mathrm{B}}
$$

if the dependence of $\underline{\underline{H}}_{B}$ with $\underline{\underline{W}}_{B}$ is neglected during the computation of the minimization. However, this type of solution is generally inaccurate and non physical (i.e., highly oscillating solutions with values greater than 1 or less than 0 ). This is due to the fact that the mathematical problem is both ill-posed and ill-conditioned. ${ }^{30,31}$ It is then possible to use regularized algorithms that include constraints on the solution. It consists in minimizing ${ }^{30,31}$

$$
\mathscr{T}_{\lambda}\left(\underline{\hat{W}}_{\mathrm{B}}\right)=\varepsilon^{2}\left(\underline{\mathrm{m}}_{\mathrm{B}}, \underline{\hat{W}}_{\mathrm{B}}\right)+\lambda \mathscr{R}\left(\underline{\hat{W}}_{\mathrm{B}}, \underline{\mathrm{W}}_{\mathrm{B}}^{\infty}\right)
$$

where $\mathscr{R}$ is a regularization functional based on some a priori knowledge on the real solution, $\lambda$ the regularization parameter that has to be determined and $\mathrm{W}_{\mathrm{B}}^{\infty}(Y)$ is an a priori reference concentration profile whose values at the measurement points $d_{i}$ are set in the vector $\underline{\mathrm{W}}_{\mathrm{B}}^{\infty}$. This reference may be the concentration profile obtained with a constant diffusion coefficient first evaluated on the measurement points and corrected with Eq. (31) or the measurements themselves. The most basic regularization functionals are quadratic and the best choice in the present cases, when considering the typical (smooth) concentration curves, leads us to minimize the roughness of the solution via its second order derivatives ${ }^{32,33}$ by using the so-called $L_{2}{ }^{34,31}$ (i.e., quadratic) regularizing functional

$$
\begin{aligned}
\mathscr{R}\left(\underline{\mathrm{W}}_{\mathrm{B}}, \underline{\mathrm{W}}_{\mathrm{B}}^{\infty}\right) & =\sum_{i=1}^{N}\left(\left.\frac{\partial^{2} \hat{\mathrm{W}}_{\mathrm{B}}}{\partial Y^{2}}\right|_{i}-\left.\frac{\partial^{2} \mathrm{~W}_{\mathrm{B}}^{\infty}}{\partial Y^{2}}\right|_{i}\right)^{2}, \\
& =\left(\underline{\hat{\mathrm{W}}}_{\mathrm{B}}-\underline{\mathrm{W}}_{\mathrm{B}}^{\infty}\right) \cdot \underline{\underline{\mathrm{D}}}_{2}{ }^{t} \cdot \underline{\underline{\mathrm{D}}}_{2} \cdot\left(\underline{\hat{\mathrm{W}}}_{\mathrm{B}}-\underline{\mathrm{W}}_{\mathrm{B}}^{\infty}\right),
\end{aligned}
$$

where $\underline{\underline{D}}_{2}$ is the second order finite differences operator (including variable discretization step $\Delta_{i}$, see Appendix B, and symmetric boundary values). If the dependence of $\underline{\underline{H}}$ with $\underline{\mathrm{W}}$ is neglected during the minimization steps, then it yields the following estimate for the reconstructed profile

$$
\underline{\hat{W}}_{\mathrm{B}}(\lambda)=\left(\underline{\underline{\mathrm{H}}}_{\mathrm{B}}^{t} \cdot \underline{\underline{\mathrm{H}}}_{\mathrm{B}}+\lambda \underline{\underline{D}}_{2}^{t} \cdot \underline{\underline{D}}_{2}\right)^{-1} \cdot\left(\underline{\underline{\mathrm{H}}}_{\mathrm{B}}^{t} \cdot \underline{\underline{m}}_{\mathrm{B}}+\lambda \underline{\underline{D}}_{2}^{t} \cdot \underline{\underline{D}}_{2} \cdot \underline{\mathrm{W}}_{\mathrm{B}}^{\infty}\right) \text {. }
$$

Matrix $\underline{\underline{D}}_{2}$ can be replaced by another differentiate order matrix or a combination of these different matrices. ${ }^{32,33}$ The choice must be made with the a priori knowledge on the solution. ${ }^{35,33,31}$ This last equation shows the effect of $\underline{\underline{D}}_{2}$ in the deconvolution process; in addition to incorporating prior knowledge on the solution (right-hand term in Eq. (40)), it increases the low eigenvalues of $\underline{\mathrm{H}}$ away from zero while leaving the high values unchanged (corrected left hand-term in Eq. (40)). This avoids ill-conditioned matrices for which the inversion may be numerically inaccurate. For the choice of the reference concentration profile, several computations show, in the present case, that $\underline{\mathrm{W}}_{\mathrm{B}}^{\infty}=\underline{0}$, i.e., no particular reference curve, appears to be a good choice. One major difficulty consists in determining the best value of the regularization parameter $\lambda$ 
that ensures that $\underline{\hat{W}}_{\mathrm{B}}(\lambda)$ is as close as possible to the true profile $\underline{\mathrm{W}}_{\mathrm{B}}$. Plotting $\mathscr{R}\left(\underline{\mathrm{W}}_{\mathrm{B}}(\lambda)\right)$ vs. $\varepsilon^{2}\left(\underline{m}_{B}, \underline{\hat{W}}_{B}(\lambda)\right)$, when the minimum of the functional $\mathscr{T}_{\lambda}$ is reached, for different values of $\lambda$ leads to an L-shaped curve (the so-called 'L-curve') where the optimal value for $\lambda$ is reached at the L-corner. ${ }^{36,37}$ The two extreme branches of the L-curve correspond to $\lambda=0$ (Eq. (37) reduces to the ill-conditioned problem with Eq. (35)) that may lead to a solution with a perfect agreement with the data (i.e., the predicted $k$-ratios agree as well as possible with the measurements $m$ ) and $\lambda \rightarrow \infty$ (i.e., the solution perfectly agrees with the a priori knowledge defined by the minimum of the regularization function). One practical way of finding $\lambda$ is to use the Generalized Cross Validation (GCV), ${ }^{35,33,31}$ which is based on finding $\lambda$ that minimizes the error between the measurements $\underline{m}_{B}$ and any reconstructed value of the measurement, $\underline{m}_{B}^{l}$, from the estimate $\underline{\hat{W}}_{\mathrm{B}}^{l}(\lambda)$ obtained by removing the $l^{\text {th }}$ point, $\mathrm{m}_{\mathrm{B}}^{l}$, from $\underline{\mathrm{m}}_{\mathrm{B}}$. This procedure consists in estimating $\lambda$ by minimizing ${ }^{33,31}$

$$
V(\lambda)=\frac{N\left(\left(\underline{\underline{\mathrm{l}}}-\underline{\underline{\mathrm{J}}}_{\mathrm{B}}(\lambda)\right) \cdot \underline{\mathrm{m}}_{\mathrm{B}}\right)^{t} \cdot\left(\left(\underline{\underline{\mathrm{l}}}-\underline{\underline{\mathrm{J}}}_{\mathrm{B}}(\lambda)\right) \cdot \underline{\mathrm{m}}_{\mathrm{B}}\right)}{\operatorname{tr}^{2}\left(\underline{\underline{\mathrm{l}}}-\underline{\underline{\mathrm{J}}}_{\mathrm{B}}(\lambda)\right)},
$$

where

$$
\underline{\underline{J}}_{\mathrm{B}}(\lambda)=\underline{\underline{\mathrm{H}}}_{\mathrm{B}} \cdot\left(\underline{\underline{\mathrm{H}}}_{\mathrm{B}}{ }^{t} \cdot \underline{\underline{\mathrm{H}}}_{\mathrm{B}}+\lambda \underline{\underline{\mathrm{D}}}_{2}^{t} \cdot \underline{\underline{\mathrm{D}}}_{2}\right)^{-1} \cdot \underline{\underline{\mathrm{H}}}_{\mathrm{B}}{ }^{t} \cdot
$$

This way of deconvoluting the signal, i.e., by minimizing the functional defined by Eq. (37) with the so-called regularized $L_{2}$-function ${ }^{34,31}$ (Eq. (38)) and the previous optimal value of $\lambda$, is usually referred to as ' $L_{2}$ deconvolution' and leads to acceptable solutions in classical cases (when the average effect is not too important, i.e., $\sqrt{2 D t} / \sigma \geq 1$, see Eq. (31)). However, in some extreme cases (when the average effect is important, i.e., $\sqrt{2 D t} / \sigma \leq 1$ ), this regularized functional is unable to avoid oscillations in the solution. An improvement is obtained by allowing for discontinuities at $l$ points of the deconvoluted curves. ${ }^{34,31}$ For these points $\lambda$ is reduced by a scale factor or set to zero. However, this includes new unknowns, i.e., the indices corresponding to the position of the discontinuities, that are not very easy to handle. A similar (but weak) 'effect', and simplified procedure, are obtained by using a non-quadratic regularized functional, ${ }^{34}$ called $L_{20}$

$$
\mathscr{R}\left(\underline{\hat{\mathrm{W}}}_{\mathrm{B}}, s\right)=\sum_{i=1}^{N} \Phi\left(\left.\frac{\partial^{2} \hat{\mathrm{W}}_{\mathrm{B}}}{\partial Y^{2}}\right|_{i}, s\right)
$$

with

$$
\Phi(u, s)=\frac{s^{2} u^{2}}{s^{2}+u^{2}}
$$

where $s$ is a threshold for the second derivative, i.e., when $|u| \leq s$ the regularized functional has a quadratic behavior (it reduces to the $L_{2}$ functional) whereas $|u|>s$ leads to an asymptotically constant function that allows one to recover, to a certain degree, some (real) strong discontinuities in the diffusion profile (i.e., when $\sqrt{2 D t} / \sigma \ll 1$ ). The corresponding functional to be minimized will be called ' $L_{20}$ deconvolution' in the following section. The value of the regularization parameter $\lambda$ is more difficult to determine in this case. We used a simplified method based on the experimental fact that most of the terms in Eq. (43) are such that $\Phi(u, s) \sim u^{2}$ (i.e., strong discontinuities occur only for few points). Consequently, for most of the points, $\lambda$ can be obtained by the GCV method (Eq. (41)). Since it gives very satisfactory results (see Section 4.3), a unique regularization parameter equal to that obtained by the previous GCV method with $\underline{\underline{H}}_{B}$ computed with $\underline{\hat{W}}_{B}=\underline{m}_{B}$ is chosen. The threshold $s$ has to be fixed or set as 
an unknown of the problem. Since it corresponds to a threshold for the second order derivative that must allow for strong discontinuities, it is linked to the ratio between the average diffusion length, $\sqrt{2\langle D\rangle t}$, and the standard deviation of the lateral X-ray distribution, i.e., $\sigma$ if the lateral $\mathrm{X}$-ray distribution is modeled by the Gaussian $g_{\sigma}$ (see Section 2). If $\sqrt{2\langle D\rangle t} / \sigma \rightarrow \infty$, then no strong discontinuities (at the considered scale) occur and $s \rightarrow \infty$ (i.e., the $L_{20}$ functional is then equivalent to the $L_{2}$ smoothing criterion). Conversely, the case $\sqrt{2\langle D\rangle t} / \sigma \rightarrow 0$ requires a threshold $s$ that is linked with the discretization step $\Delta_{i}$ (see Appendix B). A discretized weight fraction curve when no diffusion has occurred (i.e., $\sqrt{2\langle D\rangle t} / \sigma=0$ ) has indeed a maximum second order derivative equal to $1 / \min _{i}\left(\Delta_{i}\right)$, so that $s$ may be expressed as

$$
s=\left(1+\frac{\sqrt{2\langle D\rangle t}}{\sigma}\right)^{a} \frac{\mathrm{c}_{s}}{\min _{i}\left(\Delta_{i}\right)},
$$

where the exponent $a$ and the coefficient $\mathrm{c}_{s}$ must be determined. The values $a=2$ and $\mathrm{c}_{s}=1$ give acceptable results in the cases described hereafter even if a more detailed study should be performed. Note that the left-hand term between parentheses can be conveniently replaced by $\sqrt{2 D^{*} t} / \sigma$ where $D^{*}$ is the apparent (constant) diffusion coefficient obtained by fitting a weight fraction curve on the raw measurements.

Note finally that other regularization functionals exist and one of the most popular is based on the Maximum Entropy Principle ${ }^{38}$ that is well-adapted for signals with bright spots on a dark background and can yield very good results for EPMA depth profiles deconvolution of layered samples..$^{39,40,41,42}$

\subsection{Implementations, validations and results}

The $L_{2}$ deconvolution (based on Eq. (40)) is implemented by using basic subroutines for matrix inversion and one dimensional minimum search ${ }^{43}$ for $\lambda$ (Eq. (41)). Since $\underline{\mathrm{H}}_{\mathrm{B}}$ depends on $\underline{\mathrm{W}}_{\mathrm{B}}$, the algorithm is carried out several times. For each step, the transfer matrix is set to be concentration-independent and is computed with the deconvoluted weight fraction curve obtained at the previous step (the first step is initialized by using $\underline{\underline{H}}_{B}\left(\underline{m}_{B}\right)$ ). This procedure is repeated until the weight fraction profiles converge (i.e., mean square error between the previous deconvoluted profile and the new one is less than or equal to a prescribed threshold). The $L_{20}$ deconvolution (based on Eqs. (37) and (43)) uses a non-convex regularized functional and needs specific minimization algorithms. The simulated annealing procedure ${ }^{44}$ is chosen. A modified subroutine ${ }^{45}$ for the simulated annealing method is implemented. This way of minimizing the criterion is all the more interesting for the present non-linear system since it allows the transfer matrix $\underline{\mathrm{H}}_{\mathrm{B}}$ to be updated continuously during the minimization. Note that $\varepsilon\left(\underline{m}_{B}, \underline{\hat{W}}_{B}\right)$ is no longer convex in this case and the simulated annealing algorithm is all the more necessary. Unfortunately, this subroutine requires control parameters that must be tuned for the present utilization. The optimal control parameters (see an example in Table 1) are not very easy to obtain and need several tests ${ }^{44}$ on a reference deconvolution problem for example (e.g., the generalized case of Fig. 11, see next paragraph). This must be carried out with care as ill-chosen parameters lead to inaccurate (or incomplete) deconvolution. This leads to a not completely automated and difficult deconvolution method since the simulated annealing parameters (especially $T_{0}$ and $r_{T},{ }^{44,45}$ see Table 1 ) must be found and checked for each problem. In the present cases the values of Table 1 have been used successfully. Furthermore, note that the deconvoluted weight fraction point $\hat{W}_{\mathrm{B}}^{i}$ are only scanned inside the $[0,1]$ range with this 
minimization algorithm that enables one to reduce the possible number of solutions and could artificially improve the deconvolution.

The two deconvolution methods and their implementation are first validated on simple cases when using the measured B-radiations only. Constant diffusion coefficient, $D$, curves (see Eq. (49) in Appendix A) are computed and used as the real weight fraction to compute $N \times m_{\mathrm{B}}$ 'raw' measurement points with Eqs. (32) and (33) with the absorption parameter of a $\mathrm{Ni}(\mathrm{A}) / \mathrm{Cu}(\mathrm{B})$ couple (for $\delta=\pi / 2$ and $\theta=40^{\circ}$ ), the X-ray distribution of these materials for $\mathrm{E}_{0}=25 \mathrm{keV}$ (see Section 2) and white noise of standard deviation $\sigma_{\mathrm{n}}=0.01$. The curves are deconvoluted with the two different methods and a weight fraction profile based on a constant diffusion coefficient, $D^{*}$, is tuned on the deconvoluted curve and compared to the prescribed coefficient $D$. The results are shown in Fig. 10 where the error made on the diffusion coefficient, $D^{*} / D-1$, for different deconvolution functionals is plotted as a function of the average effect parameter $\sqrt{2 D t} / \sigma$. The error made on the diffusion coefficient without any deconvolution is first shown: a computed error obtained with the simulated 'raw' measurement points (i.e., including all physical effects) is compared with the simplified analytical result (Eq. (31)) only accounting for the average effect. The main differences between these two curves arise from the absorption effect (that is not considered in the closed-form solution) since it can enhance the average effect (see Section 3.1). Second, the residual error made with the different deconvolution functionals when using only the B-radiation measurements, $m_{\mathrm{B}}$, is plotted. The $L_{20}$ deconvolution yields better results than the $L_{2}$ one, since it allows for some strong discontinuities (at the scale of the standard deviation of the lateral X-ray distribution) in the weight fraction curves. However, the improvement is not very important especially when considering the higher computation time. In the specific case of a $\mathrm{Ni}(\mathrm{A}) / \mathrm{Cu}(\mathrm{B})$ couple analyzed with a 'classical' EDS detector in an SEM, the A-fluorescent radiations are of the same order of magnitude as the measurement noise. The $k_{\mathrm{A}}$-ratio can thus be assumed to be fluorescent radiations free and obtained with the same technique as for the B-radiations. The measurements of the A-radiations can thus be used for the deconvolution by replacing the reduced mean square error between the calculated and the measured data involved in Eq. (37) by the complete one of Eq. (34). This yields the last curve in Fig. 10 that corresponds to the $L_{20}$ deconvolution of the previous simulated measurements when using both $m_{\mathrm{A}}$ and $m_{\mathrm{B}}$ with a similar technique for the determination of the regularized parameter $\lambda$ and the threshold $s$ as the $L_{20}$ deconvolution when using $m_{\mathrm{B}}$ only (Eqs. (41), including $\underline{\underline{H}}_{\mathrm{A}}$ and $\underline{\underline{m}}_{\mathrm{A}}$, and (45) respectively). This leads to an important improvement in the deconvolution for low $\sqrt{2 D t} / \sigma$ ratios. Note finally that the choice in the discretization step is very important as it must be sufficiently small to correctly discretize the deconvoluted weight fraction curve. If it is not, it will reduce the quality of the deconvolution since too high displacement steps of measurement (limited by the SEM spatial resolution) never allows for a reconstruction of a diffusion curve with small diffusion lengths $\sqrt{2 D t}$.

The improvement in the resolution of EPMA measurements corrected by deconvolution can be estimated by analyzing the error curves of Fig. 10 for the simplified cases used for plotting this graph. The resolution is defined by the minimum distance between two objects such that they are just resolved. In qualitative analysis a value equal to $2 \sigma$ is found. ${ }^{24}$ In Fig. 10 , the point $\sqrt{2 D t} / \sigma=2$ corresponds to the abscissa where the error on the diffusion coefficient for 'classical' EPMA (without any deconvolution) starts to increase with an offset of 0.39 . This is depicted by the right hand downward arrow (that corresponds to $0.53 \mu \mathrm{m}$ for nickel or copper materials with $\mathrm{E}_{0}=25 \mathrm{keV}$, see Section 2). The middle right downward arrow corresponds to $L_{2}$ deconvoluted EPMA and the middle left hand arrow to the $L_{20}$ case for the same offset when using $m_{\mathrm{B}}$ only. It shows that the $L_{2}$ deconvolution technique allows one to improve the 
EPMA resolution by a factor of about 4.9 (i.e., $0.11 \mu \mathrm{m}$ for a nickel or copper material with $\mathrm{E}_{0}=25 \mathrm{keV}$ ) whereas the $L_{20}$ one allows only for an improvement of about 5.6 (i.e., $95 \mathrm{~nm}$ for a nickel or copper material with $\mathrm{E}_{0}=25 \mathrm{keV}$ ) for the simplified diffusion cases used to plot Fig. 10. A great improvement up to a factor of 9.75 (i.e., $54 \mathrm{~nm}$ for a nickel or copper material with $\mathrm{E}_{0}=25 \mathrm{keV}$ ) in the resolution can be obtained if the measured A-radiations are used in the $L_{20}$ deconvolution functional (left hand arrow) showing how it is important to improve the computation of fluorescent radiations, when they are not negligible, in order to being able to include the A-radiation measurements.

The deconvolution algorithms, when using the B-radiations measurements only, are then applied to a real case of $\mathrm{Ni}(\mathrm{A}) / \mathrm{Cu}(\mathrm{B})$ diffusion, i.e., with a concentration-dependent diffusion coefficient such that $\sqrt{2\langle D\rangle t} / \sigma \simeq 0.75$. The real weight fraction curve is computed by solving the diffusion equations ${ }^{3}$ for a $550^{\circ} \mathrm{C}$ and 10 -minute diffusion with a given concentrationdependent diffusion coefficient. The latter is measured after maintaining a $\mathrm{Ni} / \mathrm{Cu}$ electroplated diffusion couple at $550^{\circ} \mathrm{C}$ for 24 hours. ${ }^{27}$ In this last case, the average effect is so weak that it can be neglected and the real weight fraction profile is obtained by classical ZAF or $\phi(z)$ correction procedures (with $\delta \approx \pi / 2, \theta \approx 29^{\circ}$ and $\mathrm{E}_{0} \approx 25 \mathrm{keV}$ ) and used to obtain the diffusion coefficient by the Boltzmann-Matano method. ${ }^{3}$ The computed 'real' weight fraction curve is then used to simulate $N \times m_{\mathrm{B}}$ 'raw' measurement points with Eqs. (32) and (33) with the absorption parameter of a $\mathrm{Ni}(\mathrm{A}) / \mathrm{Cu}(\mathrm{B})$ couple (for $\delta=\pi / 2$ and $\theta=29^{\circ}$ ), the X-ray distribution of these materials for $\mathrm{E}_{0}=25 \mathrm{keV}$ (see Section 2) and white noise of standard deviation $\sigma_{\mathrm{n}}=0.01$. These measurements are deconvoluted and the results are shown in Fig. 11(a) where the two deconvolution functionals give similar and acceptable results but with a huge difference in computation time (e.g., more than one thousand fold) that depends on the simulated annealing parameters (see Table 1). This validates the use of the $\mathrm{B}$ radiation measurements only when the average effect is such that the resolution of the deconvolution method is not reached (see Fig. 10). The change of the deconvoluted diffusion coefficient $D$ with the weight fraction can then be extracted from the deconvoluted curves by the Boltzmann-Matano method for medium values of the weight fraction since this method is not accurate for extreme values. ${ }^{3}$ The results are compared to the real diffusion coefficient ${ }^{27}$ in Fig. 11(b). The two deconvolution functionals yield the same behavior that is acceptable when compared to the real curve because the error between the real diffusion coefficients and the deconvoluted ones is less than the error arising from the measurement noise (see the scatter due to white noise in the 'raw' measurements on the diffusion coefficient in the same figure).

Figure 12 illustrates the use of the A and B-radiation measurements in the deconvolution. The 'raw' measurements are computed by using the $\mathrm{Ni}(\mathrm{A}) / \mathrm{Cu}(\mathrm{B})$ material parameters in Eqs. (32) and (33) from a given weight fraction profile obtained with a constant diffusion coefficient (see Eq. (49) in Appendix A) such that $\sqrt{2 D t} / \sigma=0.24$. Note that the absorption effect has been increased in this case (i.e., $\mu_{\mathrm{B}}^{\mathrm{A}}=15 \mu_{\mathrm{Cu}}^{\mathrm{Ni}}$ ) to increase the difficulty of the deconvolution as shown in Section 3.1 (i.e., the difference in the mass absorption coefficient of the two materials may lead to an additional 'average' effect that adds to the standard one). The limitation of the $L_{2}$ deconvolution, when using only $m_{\mathrm{B}}$ only, is clearly shown in this figure since the deconvoluted weight fraction curves oscillate with some non-physical values (i.e., outside the $[0,1]$ range). Increasing $\lambda$ to enhance the smoothing part of the functional does not solve the problem because the result becomes too smooth. Furthermore, replacing $\underline{\underline{D}}_{2}$ by $\underline{\underline{D}}_{1}$ (i.e., the first derivative matrix) or $\underline{\underline{D}}_{0}$ (i.e., the identity matrix) in Eq. (40) is not satisfactory either, because the results are then degraded by high frequency oscillations. The improvement in the quality of the deconvolution with the $L_{20}$ functional when using both $m_{\mathrm{A}}$ and $m_{\mathrm{B}}$ is clearly seen. 
A sensitivity analysis on the regularization parameter $\lambda$ and the second order derivative threshold $s$ (for the $L_{20}$ deconvolution only) has been performed for the deconvolution cases of Figs. 11 and 12. The results are reported in Table 2 and 3, respectively. We define the mean square error for the deconvoluted weight fraction as

$$
\mathscr{E}^{2}(\lambda, s)=\sum_{i=1}^{N}\left(\hat{\mathrm{W}}_{\mathrm{B}}^{i}(\lambda, s)-\mathrm{W}_{\mathrm{B}}^{i}\right)^{2},
$$

where $\mathrm{W}_{\mathrm{B}}^{i}$ stands for the real weight fraction and $\hat{\mathrm{W}}_{\mathrm{B}}^{i}(\lambda, s)$ for the deconvoluted one for any $(\lambda, s)$ couple at the same displacement point $d_{i}$. Tables 2 and 3 contain the ratio between this error for any $(\lambda, s)$ couple and those obtained with the assumed optimal parameters, i.e., smoothing parameter obtained by the GCV method (Eq. (41)) for the $L_{2}$ and $L_{20}$ functionals and second order derivative threshold obtained with Eq. (45) for the $L_{20}$ functional. In Table 2, the sensitivity to $\lambda$ is similar and not very important for the $L_{2}$ and the $L_{20}$ deconvolution methods when using $m_{\mathrm{B}}$ only in the 'classical' case of Fig. 11. This similarity can be explained by the low sensitivity to $s$ for the $L_{20}$ deconvolution for this case as there is no strong discontinuity (see the asymptotic behavior of $\mathscr{E}^{2}$ vs. $s$ for $s>s_{\text {opt }}$ ) and the $L_{20}$ is not absolutely necessary as it can be seen in Fig. 11 where the results for $\underline{\hat{W}}_{B}$ obtained by the two deconvolution methods are very similar and close to the real weight fraction. As the two deconvolution methods are not very sensitive to $\lambda$ and $s$, the methods used to determine them (namely, the GCV method (Eq. (41)) for $\lambda$ and Eq. (45)) for $s$ ) are acceptable for the present case, i.e., when the problem is not too close to the limitation, or resolution, of the deconvolution technique. In Table 3, the sensitivity with respect to $\lambda$ and $s$ has been reported for the $L_{20}$ deconvolution when using $m_{\mathrm{A}}$ and $m_{\mathrm{B}}$ in the limit case of Fig. 12. A strong dependence to the second order derivative threshold is shown. Furthermore the error values quickly reach a plateau as well as for $s>s_{\text {opt }}$ and $s<s_{\text {opt }}$. This may arise from the specific $L_{20}$ regularization function (Eq. (43)) and a $\sqrt{2 D t} / \sigma$ ratio close to the resolution of the considered method (Fig. 10), i.e., if the threshold $s$ is too high, the deconvoluted curve is too smooth and contains too many strong discontinuities in the other case. Last, the sensitivity to the smoothing parameter $\lambda$ is not very important but the value obtained with the GCV method (Eq. (41)) seems not to be optimal since lower value leads to lower error.

Finally, when the goal of the EPMA measurements is to study diffusion, it is important to note that all the deconvoluted weight fraction curves lead to an accurate corrected position of the Matano interface, ${ }^{3}$ which corresponds to the abscissa $Y_{\mathrm{M}}$ such that $\int\left(Y-Y_{\mathrm{M}}\right) \mathrm{dW}_{\mathrm{B}}=0$. The position of this interface, which is crucially important in diffusion analyzes, ${ }^{3}$ is shifted by the average and absorption effects as shown in Fig. 12 where the downward arrow indicates the position of the Matano interface for the 'raw' measurements whereas the real Matano interface is located at $Y=0$.

\section{Conclusions}

The average effect that occurs in EDS or WDS measurements of concentration profiles is mainly described herein. It is caused by the lateral distribution of the emitted X-rays for which a model for medium to heavy materials has been proposed and compared to its 'classical' Gaussian approximation. The resulting average may have a non-negligible influence on 
the evaluation of interdiffusion coefficients when the diffusion length is of the same order of magnitude as the range of the lateral distribution of the X-rays.

When a diffusion couple made of two materials with close atomic numbers is considered, some geometrical effects associated with the average effect can occur, namely, the position of the spectrometer with respect to the concentration gradient and the non-perpendicularity of the initial interface between the two diffusing species with respect to the analyzed surface. All these effects associated with the absorption effect lead to overestimate the real diffusion coefficients and do not allow for an accurate evaluation of the position of the Matano interface. ${ }^{3}$ This study completes the effect of the fluorescence radiations on the apparent concentration profiles that has been carried out elsewhere. ${ }^{8}$

Some general deconvolution algorithms for close atomic number materials have been proposed and evaluated. They allow us to improve the EPMA resolution by a factor 5 if the B-radiations are only considered. An improvement of a factor 10 can be expected by including the A-radiations too in the analyzes. The computation of the fluorescent A-radiations is generally needed; yet faster computation algorithms are needed. These deconvolution methods may be extended for any materials if the variation of $\phi(z)$ with the weight fraction is known. Up to now, this remains a difficult task that is not completely solved analytically but some ideas, like computing each of the basic parameters involved in $\phi(z)$ with an average atomic number evaluated with an appropriate weighting law, ${ }^{46,47}$ have been useful in some cases. The techniques used to determine the deconvolution parameters, $\lambda$ and $s$, have been validated by a sensitivity analysis when the average effect is such that the resolution of the deconvolution method is not reached. However, the determination of other critical parameters, such as noise distribution, should be improved by using fully Bayesian approaches. ${ }^{30,31}$

Last, the present method for measuring concentration profiles on the submicrometre scale with EPMA must be compared to depth profiling techniques such as sputter depth profiling, ${ }^{39,40,41}$ dimple grinding profiling ${ }^{42}$ or energy variation methods ${ }^{46,47}$ that have been applied to determine the concentration and thickness of the layers of multilayered samples. The latter is interesting since it is not destructive but it is limited by the electron depth range in the sample and the number of measurement points (itself limited by the range and available values in primary electron beam acceleration voltages), the sputter depth profiling (and dimple grinding technique, which is similar) is one alternative method since it allows the user to measure deeper concentrations with improved accuracy but it is a little bit destructive. The present method is also destructive, if the diffusion couple materials analyzed is a layered sample, but adapted to diffusion profile analysis on a long spatial range. Furthermore, it can be generalized in 2D situations (e.g., similar to image deconvolution ${ }^{33,31}$ ) to improve X-ray map resolution.

Acknowledgments. The authors wish to thank Prof. J.-P. Barbot of SATIE (ENS de Cachan, France) and Prof. G. Demoment of LSS (Supélec, Orsay, France) for their helpful discussions on deconvolution techniques and Dr M. Reginatto of EML (New York, USA) for providing a copy of the deconvolution algorithm MAXED. 


\section{A A simplified convolution analysis}

In the forthcoming developments, the geometrical angle between the direction of the concentration gradient and the spectrometer, $\delta$, is assumed to be equal to $\pi / 2$. For a concentrationindependent diffusion coefficient $D$ and a given temperature $T$, the weight fraction profile for the element $X$ and for a diffusion time $t, \mathrm{~W}_{X}(Y, t)$, can be expressed by using the Fourier transform $\mathrm{F}$ of the diffusion equation ${ }^{3}$

$$
\begin{cases}\frac{\partial \mathrm{W}_{X}}{\partial t} & =D \frac{\partial^{2} \mathrm{~W}_{X}}{\partial Y^{2}} \\ \mathrm{~W}_{X}(Y, t=0) & =\mathrm{W}_{X}^{0}(Y)\end{cases}
$$

so that

$$
\mathrm{W}_{X}(Y, t)=\mathrm{F}^{-1}\left[\mathrm{~F}\left(\mathrm{~W}_{X}^{0}(Y)\right) \exp \left(-\omega^{2} D t\right)\right],
$$

and when the initial concentration is equal to a step function at $Y=0$, it leads to

$$
\mathrm{W}_{X}(Y, t)=\frac{1}{2}\left[1+\operatorname{erf}\left(\frac{Y}{2 \sqrt{D t}}\right)\right] .
$$

Since the measured concentration profile is equal to the $k$-ratio $\left(i . e ., k_{X}(d)=\mathrm{I}_{X}(d) / \mathrm{I}_{(X)}\right.$, see Eqs. (1), (8) and (9) in Section 1), one has

$$
k_{X}(d)=\frac{\int_{y=-\infty}^{\infty} \int_{z=0}^{\infty} \mathrm{W}_{X}(y+d) g_{\sigma}(y) \phi(z) \exp \left(-\mu_{X}^{\mathrm{AB}} \rho_{\mathrm{AB}} \frac{z}{\sin (\theta)}\right) \mathrm{d} y \mathrm{~d} z}{\int_{y=-\infty}^{\infty} \int_{z=0}^{\infty} g_{\sigma}(y) \phi(z) \exp \left(-\mu_{X}^{X} \rho_{X} \frac{z}{\sin (\theta)}\right) \mathrm{d} y \mathrm{~d} z},
$$

where the simplified Gaussian expression, $g_{\sigma}$, has been used for lateral X-ray distribution (see Eqs. (10) and (19) in Section 2). If we assume that $\mu_{X}^{\mathrm{AB}} \rho_{\mathrm{AB}} \sim \mu_{X}^{X} \rho_{X}$ for any $\mathrm{W}_{X}$, the integrations over $y$ and $z$ can be separated and the integration over $z$ of the numerator is equal to that of the denominator and they cancel out. This amounts to neglecting the absorption effect. Equation (50) can be rewritten as

$$
k_{X}(d)=\frac{\int_{y=-\infty}^{\infty} \mathrm{W}_{X}(y+d) g_{\sigma}(y) \mathrm{d} y}{\int_{y=-\infty}^{\infty} g_{\sigma}(y) \mathrm{d} y}=\int_{y=-\infty}^{\infty} \mathrm{W}_{X}(y+d) g_{\sigma}(y) \mathrm{d} y,
$$

since $\int_{y=-\infty}^{\infty} g_{\sigma}(y) \mathrm{d} y=1$ by definition of the Gaussian distribution. The previous equation shows that $k_{X}(d)$ is equal to the convolution product between the concentration profile and the lateral distribution of the $\mathrm{X}$-ray emission. It can be simplified by invoking the Fourier transform and using Eq. (19) of Section 2

$$
\begin{aligned}
k_{X}(d) & =\int_{y=-\infty}^{\infty} \mathrm{W}_{X}(y+d) \frac{1}{\sqrt{2 \pi} \sigma} \exp \left(-\frac{y^{2}}{2 \sigma^{2}}\right) \mathrm{d} y \\
& =\int_{Y=-\infty}^{\infty} \mathrm{W}_{X}(Y) \frac{1}{\sqrt{2 \pi} \sigma} \exp \left(-\frac{(Y-d)^{2}}{2 \sigma^{2}}\right) \mathrm{d} Y \\
& =\mathrm{F}^{-1}\left[\mathrm{~F}\left(\mathrm{~W}_{X}(Y)\right) \exp \left(-\frac{\omega^{2} \sigma^{2}}{2}\right)\right] .
\end{aligned}
$$


This last expression is similar to Eq. (48) and corresponds to the Fourier transform of a convolution product. By Fourier transforming $\mathrm{W}_{X}(y, t)$ given in Eq. (48), Eq. (54) can be rewritten as

$$
\begin{aligned}
k_{X}(d) & =\mathrm{F}^{-1}\left[\mathrm{~F}\left(\mathrm{~W}_{X}^{0}(Y)\right) \exp \left(-\omega^{2} D t\right) \exp \left(-\frac{\omega^{2} \sigma^{2}}{2}\right)\right], \\
& =\mathrm{F}^{-1}\left[\mathrm{~F}\left(\mathrm{~W}_{X}^{0}(Y)\right) \exp \left(-\omega^{2} D^{*} t\right)\right], \\
& =\int_{Y=-\infty}^{\infty} \mathrm{W}_{X}^{0}(Y) \frac{1}{2 \sqrt{\pi} D^{*} t} \exp \left(-\frac{(Y-d)^{2}}{4 D^{*} t}\right) \mathrm{d} Y,
\end{aligned}
$$

with

$$
D^{*}=D+\sigma^{2} / 2 t
$$

If the initial concentration profile is equal to a step function, then a similar expression as Eq. (49) is obtained

$$
k_{X}(d)=\frac{1}{2}\left[1+\operatorname{erf}\left(\frac{d}{2 \sqrt{D^{*} t}}\right)\right],
$$

where the apparent diffusion coefficient $D^{*}$ is different from the true diffusion coefficient $D$ if $\sigma^{2} / 2 t$ is not negligible compared to $D$. Equation (58) describes the average effect of the Xray characteristic emission distribution (under the specified assumptions on the diffusion and absorption coefficients) and has been obtained by other authors following different routes. ${ }^{28,29}$ Note that the previous derivation is exact for a perfect interface even for strong concentrationdependent coefficients if $\delta=\pi / 2$. The above results can be used to identify $\sigma$ from EPMA measurements ${ }^{26,8}$ as described by Eq. (20) in Section 2. 


\section{B Expression of the transfer matrix $\underline{\underline{H}}$}

This appendix aims at determining the expression of the second order transfer matrix $\underline{\underline{\mathrm{H}}}$ defined by

$$
\mathrm{k}_{X}^{i}=\sum_{j=1}^{N} \mathrm{H}_{X}^{i j}\left(\underline{\mathrm{W}}_{X}\right) \mathrm{W}_{X}^{j} .
$$

It consists in discrete values of the absorption corrected emission function $g_{\sigma}(y) \phi(z)$. It is restricted to the case $\delta=\pi / 2$, with the Packwood and Brown depth distribution function $\phi(z)^{17,18}$ and a Gaussian lateral distribution $g_{\sigma}(y)$ (see Section 2). Equation (60) initially expressed as (see Eqs. (1), (8) and (9) in Section 1)

$$
\begin{aligned}
\mathrm{k}_{X}^{i} & =k_{X}\left(d=y_{i}\right)=\frac{\mathrm{I}_{X}\left(y_{i}\right)}{\mathrm{I}_{(X)}} \\
& =\int_{y=-\infty}^{\infty} \int_{z=0}^{\infty} \mathrm{W}_{X}\left(y+y_{i}\right) \frac{g_{\sigma}(y) \phi(z) \exp \left(-\mu_{X}^{\mathrm{AB}} \rho_{\mathrm{AB}} \frac{z}{\sin (\theta)}\right)}{\mathrm{I}_{(X)}^{\prime}} \mathrm{d} y \mathrm{~d} z,
\end{aligned}
$$

with

$$
\mathrm{I}_{(X)}^{\prime}=\int_{y=-\infty}^{\infty} \int_{z=0}^{\infty} g_{\sigma}(y) \phi(z) \exp \left(-\mu_{X}^{X} \rho_{X} \frac{z}{\sin (\theta)}\right) \mathrm{d} y \mathrm{~d} z
$$

corresponds to the intensity measured by the spectrometer for a pure material divided by the parameter $\Delta_{\mathrm{I}}$ (containing the solid angle of the detector and other factors, see Section 1). If $\mathrm{W}_{X}$ is assumed to be stepwise constant, i.e., $\mathrm{W}_{X}(y)=\mathrm{W}_{X}^{i}$ for $y_{i}-\Delta_{i-1} / 2 \leq y<y_{i}+\Delta_{i} / 2$ with $\Delta_{i}=y_{i+1}-y_{i}$ and the concentration profile is considered to be constant for $-\infty<y<y_{1}$ and $y_{N}<y<+\infty$ (see Fig. 13), then the previous integrations can be replaced by a summation of sub-integrations over each interval $\left[y_{i}-\Delta_{i-1} / 2, y_{i}+\Delta_{i} / 2\right]$. Replacing the integration variable $y$ by $\eta=y+y_{i}$ leads to

$$
\mathrm{k}_{X}^{i}=\frac{\pi \sigma}{2 \sqrt{2} \alpha \rho_{X} \mathrm{I}_{(X)}^{\prime}} \sum_{j} \mathrm{~W}_{X}^{j} \underbrace{\left[\gamma \mathscr{G}\left(\mu_{1}^{j}\right)-\left(\gamma-\phi_{0}\right) \mathscr{G}\left(\mu_{2}^{j}\right)\right]}_{\text {integration over } z} \overbrace{\left[\operatorname{erf}\left(\frac{y}{\sqrt{2} \sigma}\right)\right]_{y_{1}^{i, j}}^{y_{2}^{i, j}},}^{\text {integration over } \eta},
$$

where $\mathscr{G}(\xi)=\exp \left(\xi^{2}\right) \operatorname{erf}(\xi)$, and

$$
\begin{aligned}
\mu_{1}^{j} & =\frac{(\mu \rho)_{X}^{j}}{2 \alpha \rho_{X} \sin (\theta)} \\
\mu_{2}^{j} & =\frac{\beta \rho_{X} \sin (\theta)+(\mu \rho)_{X}^{j}}{2 \alpha \rho_{X} \sin (\theta)} \\
y_{1}^{i, j} & =y_{j}-y_{i}-\frac{\Delta_{j-1}}{2} \\
y_{2}^{i, j} & =y_{j}-y_{i}+\frac{\Delta_{j}}{2}
\end{aligned}
$$

with (see Eqs. (5) and (6) in Section 1)

$$
(\mu \rho)_{X}^{j}=\left(\mathrm{W}_{X}^{j} \mu_{X}^{X}+\left(1-\mathrm{W}_{X}^{j}\right) \mu_{X}^{\bar{X}}\right)\left(\mathrm{W}_{X}^{j} \rho_{X}^{-1}+\left(1-\mathrm{W}_{X}^{j}\right) \rho_{\bar{X}}^{-1}\right)^{-1} .
$$


Equation (63) is not satisfied for $j=1$ and $j=N$ since the right-hand side terms (i.e., integration over $\eta$ ) must be replaced by

$$
\begin{cases}1+\operatorname{erf}\left(\frac{y_{1}-y_{i}+\Delta_{1} / 2}{\sqrt{2} \sigma}\right) & \text { if } j=1 \\ 1-\operatorname{erf}\left(\frac{y_{N}-y_{i}+\Delta_{N-1} / 2}{\sqrt{2} \sigma}\right) & \text { if } j=N\end{cases}
$$

since $\Delta_{0}$ and $\Delta_{N}$ are assumed to tend to infinity (Fig. 13). The components of the transfer matrix are expressed by

$$
\mathrm{H}^{i j}=\Xi_{j} \cdot \begin{cases}\operatorname{erf}\left(\frac{y_{2}^{i, j}}{\sqrt{2} \sigma}\right)-\operatorname{erf}\left(\frac{y_{1}^{i, j}}{\sqrt{2} \sigma}\right) & \text { for } 2 \leq j \leq N-1, \\ 1+\operatorname{erf}\left(\frac{y_{1}-y_{i}+\Delta_{1} / 2}{\sqrt{2} \sigma}\right) & \text { for } j=1, \\ 1-\operatorname{erf}\left(\frac{y_{N}-y_{i}+\Delta_{N-1} / 2}{\sqrt{2} \sigma}\right) & \text { for } j=N\end{cases}
$$

with

$$
\Xi_{j}=\frac{\pi \sigma}{2 \sqrt{2} \alpha \rho_{X} \mathrm{I}_{(\mathrm{X})}^{\prime}}\left[\gamma \mathscr{G}\left(\mu_{1}^{j}\right)-\left(\gamma-\phi_{0}\right) \mathscr{G}\left(\mu_{2}^{j}\right)\right] .
$$




\section{References}

1. Castaing R. Ph. D. Thesis, University of Paris, 1951. O.N.E.R.A. Publication no. 55, 1952.

2. Reed SJB. Matrix Corrections in Practice. In Electron Microprobe Analysis $-2^{\text {nd }}$ Ed. Cambridge University Press: Cambridge, 1993; 262-273.

3. Philibert J. Atoms Movement and Mass Transport in Solids. Les éditions de Physique: Les Ulis, 1991.

4. Heinrich KFJ. Lateral Distribution of X-Ray Generation. In Electron Beam X-Ray Microanalysis. Van Nostrand Reinhold Company: New York, 1981; 422-424.

5. Duncumb P. Improved Resolution with the X-Ray Scanning Microanalyser. In $2^{\text {nd }}$ International Symposium on X-Ray Microscopy and X-Ray Microanalysis, Engström A, Cosslett V, Pattee HH (eds). Elsevier: Amsterdam, 1960; 365-371.

6. Goldstein JI, Newbury DE, Echlin P, Joy DC, Fiori C, Lifshin E. Electron-Beam-Specimen Interactions. In Scanning Electron Microscopy and X-Ray Microanalysis. Plenum Press: New York, 1981; 53-122.

7. Reed SJB. X-Ray Generation and Stopping Power. In Electron Microprobe Analysis $-2^{\text {nd }}$ Ed. Cambridge University Press: Cambridge, 1993; 186-201.

8. Arnould O, Hild F. Mikrochim. Acta, 2002; 139(1-4): 3-10.

9. Reed SJB, Long JVP. Electron-Probe Measurements near Phase Boundaries. In $3^{\text {rd }}$ International Conference on X-Ray Optics and Microanalysis, Pattee HH, Cosslett VE, Engström A (eds). Academic Press: New York, 1963; 317-327.

10. Maurice F, Seguin R, Henoc J. Phénomènes de fluorescence dans les couples de diffusion. In $4^{\text {th }}$ International Conference on X-Ray Optics and Microanalysis, Castaing R, Deschamps P, Philibert J, (eds). Hermann: Paris, 1966; 357-364.

11. Henoc J, Maurice F, Zemskoff A. Phénomènes de fluorescence aux limites de phases. In $5^{\text {th }}$ International Conference on X-Ray Optics and Microanalysis, Möllenstedt G, Gaukler KH (eds). Springer: Berlin, 1969; 187-192.

12. Bastin GF, van Loo FJJ, Vosters PJC, Vrolijk JWGA. Scanning, 1983; 5: 172-183.

13. Llovet X, Valovirta E, Heikinheimo E. Mikrochim. Acta, 2000; 132: 205-212.

14. Valovirta E, Erlach S, Llovet X, Heikinheimo E. EPMA of Metal-Metal Diffusion Couples at High Temperature. In EMAS $2001-7^{\text {th }}$ European Workshop on Modern Developments and Applications in Microbeam Analysis, Heikinheimo E, Walker CT, Armigliato A (eds). European Microbeam Analysis Society, 2001; 382.

15. Reed SJB. Introduction. In Electron Microprobe Analysis $-2^{\text {nd }}$ Ed. Cambridge University Press: Cambridge, 1993; 1-12.

16. Joy DC. Monte Carlo Modeling for Electron Microscopy and Microanalysis. Oxford University Press: New York, 1995. Download site: http://web.utk.edu/ srcutk/htm/simulati.htm [January 2003]. 
17. Packwood RH, Brown JD. X-Ray Spectrom., 1981; 10(3): 138-146.

18. Brown JD, Packwood RH. X-Ray Spectrom., 1982; 11(4): 187-193.

19. Bastin GF, Heijligers HJM, van Loo FJJ. Scanning, 1986; 8: 45-67.

20. Riveros J, Castellano G. X-Ray Spectrom., 1993; 22: 3-10.

21. Numerical integration by Reed ${ }^{24}$ of: Bishop HE. Proc. Phys. Soc., 1965; 85: 855-866.

22. Barkshire I, Karduck P, Rehbach WP, Richter S. Mikrochim. Acta, 2000; 132(2-4): 113128.

23. Wittry DB. J. Appl. Phys., 1958; 29(11): 1543-1548.

24. Reed SJB. Spatial Resolution in Electron-Probe Microanalysis. In $4^{\text {th }}$ International Conference on X-Ray Optics and Microanalysis, Castaing R, Deschamps P, Philibert J (eds). Hermann: Paris, 1966; 339-349.

25. Russ JC. Energy Dispersion X-Ray Analysis on the Scanning Electron Microscope. In Energy Dispersion X-Ray Analysis: X-Ray and Electron Probe Analysis, ASTM: Philadelphia, 1971; STP 485, 154-179.

26. Arnould O, Hild F. Microscopy and Analysis, 2000; 66: 25-27 (Americas Edition), 13-15 (European Edition).

27. Arnould O, Hild F. Long-Term Life of Ni/Cu Bellows: Effect of Diffusion on Thermomechanical Properties. In Defect and Diffusion in Metals - An Annual Retrospective IV, Fisher DJ (ed). Defect and Diffusion Forum; Scitec Publications: Uetikon-Zurich (Switzerland), 2002; 203-205: 61-80.

28. Lo CC, Schuele DE. J. Appl. Phys., 1975; 46(11): 5004-5009.

29. Ganguly J, Bhattacharya RN, Chakraborty S. Am. Mineral., 1988; 73: 901-909.

30. Demoment G. IEEE Trans. Acoust. Speech. Signal Process., 1989; 37(12): 2024-2036.

31. Demoment G, Idier J, Giovannelli JF, Mohammad-Djafari A. Problèmes inverses en traitement du signal et de l'image. In Les Techniques de l'Ingénieur, 2001; TE 5235-1 TE 5235-25.

32. Cullum J. Math. Comput., 1979; 33(145): 149-170.

33. Fortier N, Demoment G., Goussard Y. J. Vis. Commun. Image Represent., 1993; 4: 157170.

34. Idier J. Regularization Tools and Model for Image and Signal Reconstruction. In $3^{\text {rd }}$ International Conference on Inverse Problems in Engineering, 1999; Website. http://www.me.ua.edu/3icipe/fin3prog.htm [January 2003].

35. Thompson AM, Brown JC, Kay JW, Titterington DM. IEEE Trans. Pattern Anal. Mach. Intell., 1991; 13(4): 326-339.

36. Hansen P. SIAM Rev., 1992; 34(4): 561-580. 
37. Gulliksson ME, Wedin PA. Optimization Tools for Inverse Problems using the Nonlinear L-Curve and A-Curve. In $3^{\text {rd }}$ International Conference on Inverse Problems in Engineering, 1999; Website. http://www.me.ua.edu/3icipe/fin3prog.htm [January 2003].

38. Gull SF, Skilling J. Theory. In Quantified Maximum Entropy - MemSys5 User's Manual. The Maximum Entropy Data Consultants Website. http://www.maxent.co.uk/ [January 2003].

39. Smith GC, Park D, Cochonneau O. J. Microsc., 1995; 178(1): 48-55.

40. Richter S, Lesch N, Karduck P. Mikrochim. Acta, 1998; [Suppl.] 15: 125-131.

41. Lesch N, Aretz A, Pidun M, Richter S, Karduck P. Mikrochim. Acta, 2000; 132(2-4): 377382.

42. Richter S, Karduck P. Developments in EPMA Depth Profiling using the Dimple Grinding Techniques. In EMAS $2001-7^{\text {th }}$ European Workshop on Modern Developments and Applications in Microbeam Analysis, Heikinheimo E, Walker CT, Armigliato A (eds). European Microbeam Analysis Society, 2001; 367.

43. Press WH, Teukolsky SA, Vetterling WT, Flannery BP. Numerical Recipes in Fortran $-2^{\text {nd }}$ Ed. Cambridge University Press: Cambridge, 1992. Website: http://www.library.cornell.edu/nr/bookfpdf.html [January 2003].

44. Goffe WL, Ferrier GD, Rogers J. J. Econom., 1994; 60: 65-99.

45. Reginatto M, Goldhagen P. MAXED, A Computer Code For the Deconvolution of Multisphere Neutron Spectrometer Data Using the Maximum Entropy Method; Report no. 595. The Environmental Measurements Laboratory Website. http://www.eml.doe.gov/publications/reports/ [January 2003].

46. Pouchou JL. Anal. Chim. Acta, 1993; 283(1): 81-97.

47. Pouchou JL. Mikrochim. Acta, 2002; 138(3-4): 133-152. 


\section{List of Figures}

1 Schematic of the entire process of $X$-ray emission in a concentration (weight fraction) profile $\mathrm{W}_{\mathrm{B}}(Y)$ for $\mathrm{A} / \mathrm{B}$ diffusion couples (with $\mathrm{Z}_{\mathrm{B}}>\mathrm{Z}_{\mathrm{A}}$ ). The change in concentration is depicted by the gray level (the darker, the richer in Belement). An electron beam is focused on a point I of the sample surface at a distance $d$ from the origin, $Y=0$, of the concentration curves $\mathrm{O}$ corresponding to the initial interface between the $\mathrm{A}$ and $\mathrm{B}$ elements with no diffusion. The spread of the electrons in the sample produces a 3D characteristic emission of $\mathrm{X}$-rays. Each point, $\mathrm{S}$ of this distribution generates A and B primary X-rays that emerge from the sample surface at $\mathrm{N}$, with a take-off angle $\theta$, to reach the spectrometer whose orientation with respect to the concentration gradient is $\delta$. It detects the $\mathrm{A}$ and $\mathrm{B} \mathrm{X}$-ray intensities, i.e., $\mathrm{I}_{\mathrm{A}}$ and $\mathrm{I}_{\mathrm{B}}$. Moreover, $\mathrm{B}$ radiations are absorbed in the material and can produce secondary excitation of A-atoms at M. The generated fluorescent A-radiations that emerge from the sample surface can reach the spectrometer that detects their intensity $\mathrm{I}_{\mathrm{A}}^{\mathrm{f}}$. . . . . 2D X-ray emission distribution in copper obtained by Monte Carlo simulations ${ }^{16}$ with an electron probe diameter of $10 \mathrm{~nm}$ and an acceleration voltage $\mathrm{E}_{0}=25 \mathrm{keV}$. Definition of the lateral X-ray distribution $\psi(y)$ and the depth one, $\phi(z)$, by integration of the 2D distribution over thin layer of thicknesses $\mathrm{d} y$ and $\mathrm{d} z$, respectively. $\phi(z)$ generally contains a transient and a Gaussian part delimited by the depth $z_{r}$ that stands for the depth where the effect of the transient function in Eq. (13) becomes negligible. For medium to heavy materials (i.e., atomic number greater than ten) and negligible primary electron beam size with respect to the whole area affected, $\psi(y)$ is assumed to be close to the part of $\phi(z)$ between $z_{r}$ and $\infty$, which is depicted by the dashed lines. . . . . . . . . .

3 (a) Change of $k_{\mathrm{B}}$-ratio vs. $d$ for different lateral $\mathrm{X}$-ray distributions fitted on EDS measurements when crossing a perfect interface of a $\mathrm{Ni}(\mathrm{A}) / \mathrm{Cu}(\mathrm{B})$ electrodeposited couple. Only a fraction of the measurement points is shown for the sake of clarity (total number: 44). (b) Corresponding lateral X-ray distributions. The derivative of the measured $k_{\mathrm{B}}$-ratio has been obtained by considering the average slope for 5 consecutive measurement points. $\left(\mathrm{E}_{0} \approx 25 \mathrm{keV}, \delta \approx\right.$

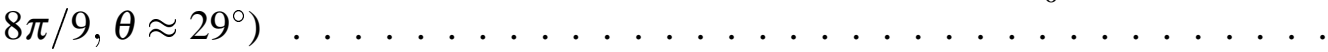

4 (a) Effect of the spectrometer angular position, $\delta$, and mass absorption coefficient, $\mu_{\mathrm{B}}^{\mathrm{A}}$, on the measured $k_{\mathrm{B}}(d)$-ratio (simulated with Eq. (21)) for a perfect interface. (b) Change of $k_{\mathrm{B}}$ for $d=0$ (i.e., primary electron beam focused on the interface) with spectrometer angle, $\delta$, for different absorption coefficients $\mu_{\mathrm{B}}^{\mathrm{A}}$ (Eq. (21)). All the other parameters correspond to a $\mathrm{Ni}(\mathrm{A}) / \mathrm{Cu}(\mathrm{B})$ couple for $\mathrm{E}_{0}=25 \mathrm{keV}, \theta=29^{\circ}$ and $\mu_{\mathrm{B}}^{\mathrm{A}^{0}}=\mu_{\mathrm{Cu}}^{\mathrm{Ni}}$.

5 (a) Comparison between the Monte Carlo computation ${ }^{16}$ of the X-ray distribution for copper $\left(\mathrm{E}_{0}=25 \mathrm{keV}\right)$ with the approximated spherical one (sphere of radius $R_{\mathrm{S}}$ centered at a depth $z_{r}$ ). Gray surfaces define the partial volumes used in Eq. (23) when crossing a perfect interface. (b) Dimensionless root mean square error between a spherical X-ray emission distribution with a depth shift $z_{r}$ (Eq. (23)) and a hemispherical distribution, i.e., $z_{r}=0$ (Eq. (26)), vs. dimensionless depth shift $z_{r} / R_{\mathrm{S}} \ldots \ldots \ldots \ldots \ldots$ 
Determination of the radius $R_{\mathrm{S}}$ of the simplified spherical X-ray distribution by fitting the curve obtained with Eq. (26) on EDS measurements when crossing a perfect $\mathrm{Ni}(\mathrm{A}) / \mathrm{Cu}(\mathrm{B})$ interface $\left(\mathrm{E}_{0} \approx 25 \mathrm{keV}, \delta \approx 8 \pi / 9, \theta \approx 29^{\circ}\right) . \ldots . . . .34$

7 (a) Simplified hemispherical X-ray emission volume and the effect of a nonperpendicular interface ( $\zeta$ is positive and $d$ negative). Gray surfaces define the volume used in Eq. (27). (b) Simulated hemispherical effect for a perfect straight interface with a misorientation angle $\zeta$ obtained with Eq. (27). . . . . .

(a) Change in the corrected coefficient of asymmetry $\left|\gamma_{1}-0.42 \zeta\right|$ (Eq. (29)) with the misorientation angle $|\zeta|$ and schematic of the $\mathrm{Ni} / \mathrm{Cu}$ tubular specimen used to determine the misorientation angle $(\sim 0.44 \pi)$ from the corrected skewness (0.82). (b) $k_{\mathrm{B}}$-ratio measurements on a $\mathrm{Ni}(\mathrm{A}) / \mathrm{Cu}(\mathrm{B})$ couple with a non-perpendicular interface ( $\zeta \approx 0.4 \pi$ obtained by optical measurement) and the corresponding simulated $k_{\mathrm{B}}$-ratio with Eq. (27) and $\zeta \approx 0.44 \pi$ obtained by the measured skewness. Only a fraction of the measurement points is shown for the sake of clarity (total number: 54$)\left(\mathrm{E}_{0} \approx 25 \mathrm{keV}, \delta=\pi / 2, \theta \approx 29^{\circ}\right) .$.

9 Apparent concentration dependence of the dimensionless diffusion coefficient due to a misoriented interface with no diffusion, $D_{\zeta}^{*} ; D_{0}^{*}$ stands for the apparent diffusion coefficient when measuring the concentration profile for a perfect interface with a hemispherical X-ray emission distribution. . . . . . . . . . .

10 Error between the real diffusion coefficient, $D$, and the raw (without deconvolution) or deconvoluted ones, $D^{*}$, obtained by different deconvolution techniques vs. the ratio between the diffusion length, $\sqrt{2 D t}$, and the standard deviation of the lateral X-ray distribution $\sigma$ that evaluates the average effect. All the curves, except the second one, are obtained by simulating raw measurements with Eqs. (32) and (33) for a constant diffusion coefficient, $D$, curve (Eq. (49)) with a white noise of standard deviation $\sigma_{\mathrm{n}}=0.01$. The obtained measurement curves are discretized. The first curve is obtained without any deconvolution by assuming an apparent constant diffusion coefficient $D^{*}$ curve (Eq. (49)). The second is a direct plot of Eq. (31). The three following curves have been obtained by assuming an apparent constant diffusion coefficient $D^{*}$ curve (Eq. (49)) on deconvoluted weight fraction curves with different functionals and measurements, i.e., the first two curves use only the B-radiation measurements, $m_{\mathrm{B}}$, in the deconvolution functional whereas the last curve uses both the $\mathrm{A}$ and $\mathrm{B}$-radiations measurements when the fluorescent A-radiations can be neglected (all material parameters are equivalent to a $\mathrm{Ni}(\mathrm{A}) / \mathrm{Cu}(\mathrm{B}) \mathrm{cou}-$ ple with $\mathrm{E}_{0}=25 \mathrm{keV}, \sigma_{\mathrm{n}}=0.01, \delta=\pi / 2, \theta=40^{\circ}$ ). The regularization parameter $\lambda$ is obtained by the GCV method (Eq. (41)) and the second order derivative threshold $s$ for the $L_{20}$ deconvolution with Eq. (45). The downward arrows correspond to the resolution obtained without deconvolution and with the $L_{2}$ and $L_{20}$ ones. The $L_{2}$ deconvolution (with $m_{\mathrm{B}}$ only) allows one to improve the EPMA resolution by a factor 4.9 and the $L_{20}$ (with $m_{\mathrm{A}}$ and $m_{\mathrm{B}}$ ) one by a factor of 9.8 for the simplified cases used to plot this graph. . . . . . . 
11 Regularized deconvolution of simulated raw B-radiations measurements (Eqs. (32) and (33)) for a real concentration-dependent diffusion coefficient of $\mathrm{Ni}(\mathrm{A}) / \mathrm{Cu}(\mathrm{B})$ electroplated diffusion couple ${ }^{27}$ at $550^{\circ} \mathrm{C}$ for 10 minutes $(\sqrt{2\langle D\rangle t} / \sigma \simeq 0.75)$ : (a) Weight fraction curves and deconvolution(s) when using the B-radiations measurements only. The two deconvolution techniques (Eqs. (38) and (43)) lead to very similar results (only the $L_{20}$ deconvolution is shown). The reconstructed measurements correspond to the $k$-ratio (Eq. (33)) obtained with the deconvoluted weight fraction profile obtained by $L_{20}$ deconvolution. Only a fraction of the points is shown for the sake of clarity (total number: 40). (b) Diffusion coefficient dependence with weight fraction in $\mathrm{Cu}(\mathrm{B})$ obtained by the Boltzmann-Matano method $^{3}$ where the derivative is computed as the average slope of 6 consecutive points. The two deconvolution functionals yield the same acceptable behavior since the error between the real diffusion coefficients and the deconvoluted ones is less than the error arising from the measurement noise depicted by the scatter in the raw diffusion coefficient $\left(\sigma_{\mathrm{n}}=0.01, \mathrm{E}_{0}=\right.$ $25 \mathrm{keV}, \delta=\pi / 2, \theta=29^{\circ}, \lambda=\lambda_{\mathrm{GCV}} \approx 24, s=s_{\mathrm{opt}} \approx 49$ (Eq. (45))). . . . . . 39

12 Regularized deconvolution of simulated raw measurements (Eqs. (32) and (33)) for a weight fraction curve with a constant diffusion coefficient (Eq. (49)) when $\sqrt{2 D t} / \sigma=0.24$. Comparison between the $L_{2}$ deconvolution when using the Bradiations measurements only (Eqs. (38)) and $L_{20}$ one when using both $\mathrm{A}$ and B-radiations measurements (Eqs. (34) and (43)). The reconstructed measurements correspond to the $k$-ratio obtained with the deconvoluted weight fraction profile obtained by $L_{20}$ deconvolution (Eq. (33)). The downward arrow corresponds to the position of the Matano interface ${ }^{3}$ for the raw measurement curve whereas its real position (determine with the real weight fraction curve) is located at $Y=0\left(\sigma_{\mathrm{n}}=0.01, \mathrm{E}_{0}=25 \mathrm{keV}, \delta=\pi / 2, \theta=29^{\circ}\right.$, material parameters correspond to a $\mathrm{Ni}(\mathrm{A}) / \mathrm{Cu}(\mathrm{B})$ couple except $\mu_{\mathrm{B}}^{\mathrm{A}}=15 \mu_{\mathrm{Cu}}^{\mathrm{Ni}}$ to increase the absorption effect, $\lambda=\lambda_{\mathrm{GCV}} \approx 47, s=s_{\mathrm{opt}} \approx 0.2$ (Eq. (45))). Only a fraction of the points (total number: 45) and the B-curves are shown for the sake of clarity . . . 40

13 Typical concentration profile and its spatial discretization with stepwise constant functions corresponding to measurement points. . . . . . . . . . . . . . . 41 


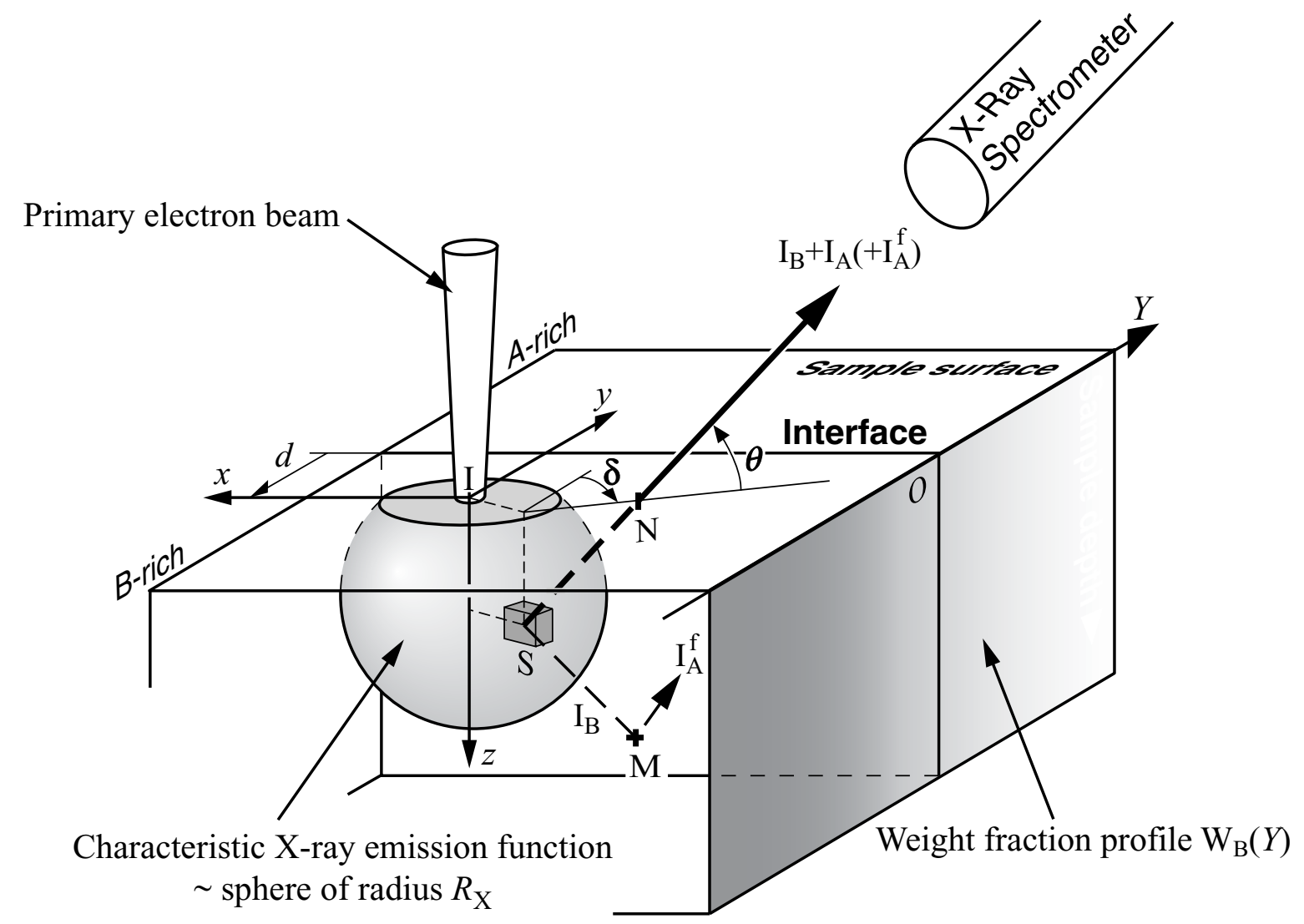

Figure 1: Schematic of the entire process of $X$-ray emission in a concentration (weight fraction) profile $\mathrm{W}_{\mathrm{B}}(Y)$ for $\mathrm{A} / \mathrm{B}$ diffusion couples (with $\mathrm{Z}_{\mathrm{B}}>\mathrm{Z}_{\mathrm{A}}$ ). The change in concentration is depicted by the gray level (the darker, the richer in B-element). An electron beam is focused on a point I of the sample surface at a distance $d$ from the origin, $Y=0$, of the concentration curves $\mathrm{O}$ corresponding to the initial interface between the $\mathrm{A}$ and $\mathrm{B}$ elements with no diffusion. The spread of the electrons in the sample produces a 3D characteristic emission of X-rays. Each point, $\mathrm{S}$ of this distribution generates $\mathrm{A}$ and $\mathrm{B}$ primary $\mathrm{X}$-rays that emerge from the sample surface at $\mathrm{N}$, with a take-off angle $\theta$, to reach the spectrometer whose orientation with respect to the concentration gradient is $\delta$. It detects the $\mathrm{A}$ and $\mathrm{B} X$-ray intensities, i.e., $\mathrm{I}_{\mathrm{A}}$ and $\mathrm{I}_{\mathrm{B}}$. Moreover, B-radiations are absorbed in the material and can produce secondary excitation of A-atoms at $\mathrm{M}$. The generated fluorescent A-radiations that emerge from the sample surface can reach the spectrometer that detects their intensity $\mathrm{I}_{\mathrm{A}}^{\mathrm{f}}$. 


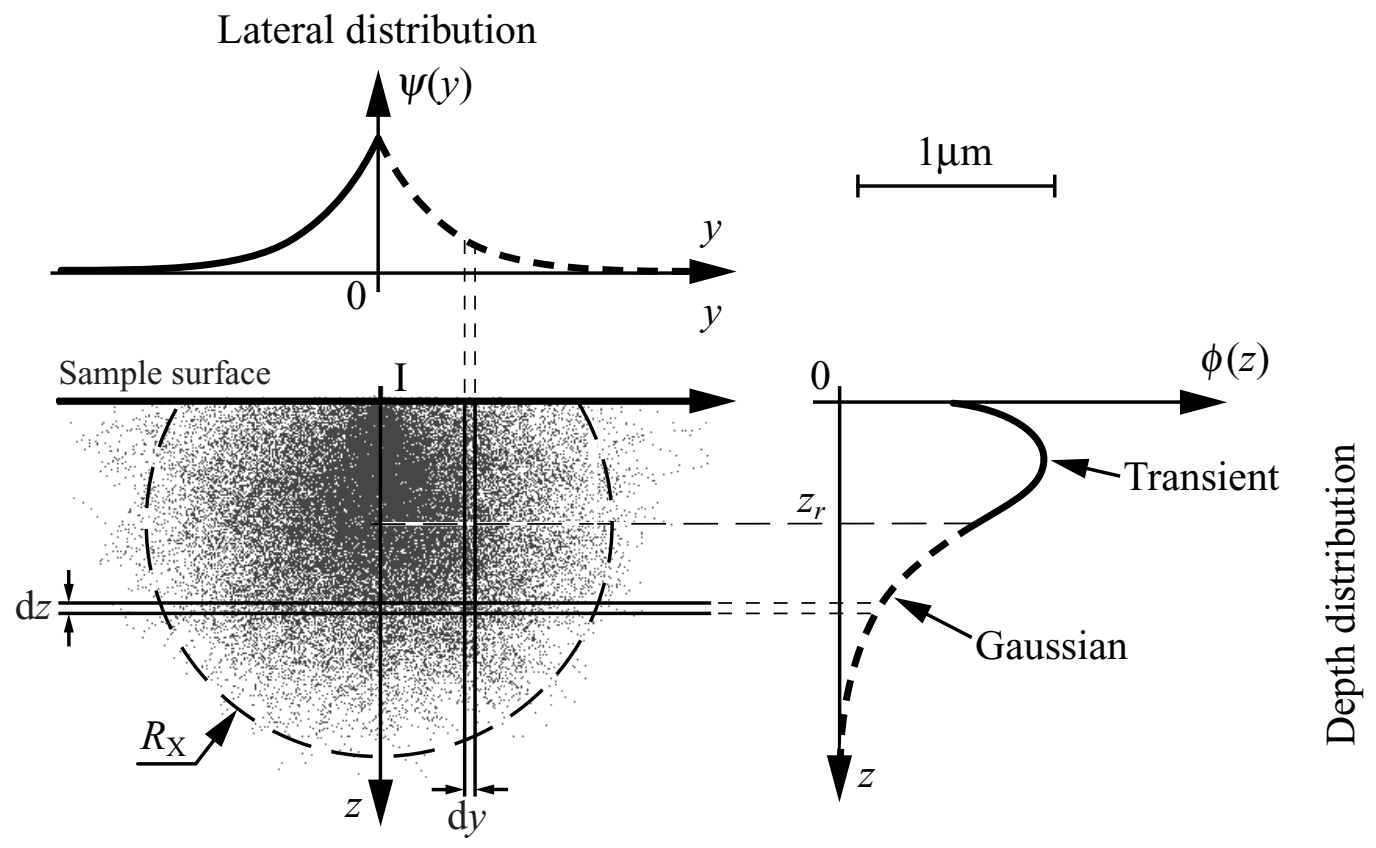

Figure 2: 2D X-ray emission distribution in copper obtained by Monte Carlo simulations ${ }^{16}$ with an electron probe diameter of $10 \mathrm{~nm}$ and an acceleration voltage $\mathrm{E}_{0}=25 \mathrm{keV}$. Definition of the lateral X-ray distribution $\psi(y)$ and the depth one, $\phi(z)$, by integration of the 2D distribution over thin layer of thicknesses $\mathrm{d} y$ and $\mathrm{d} z$, respectively. $\phi(z)$ generally contains a transient and a Gaussian part delimited by the depth $z_{r}$ that stands for the depth where the effect of the transient function in Eq. (13) becomes negligible. For medium to heavy materials (i.e., atomic number greater than ten) and negligible primary electron beam size with respect to the whole area affected, $\psi(y)$ is assumed to be close to the part of $\phi(z)$ between $z_{r}$ and $\infty$, which is depicted by the dashed lines. 


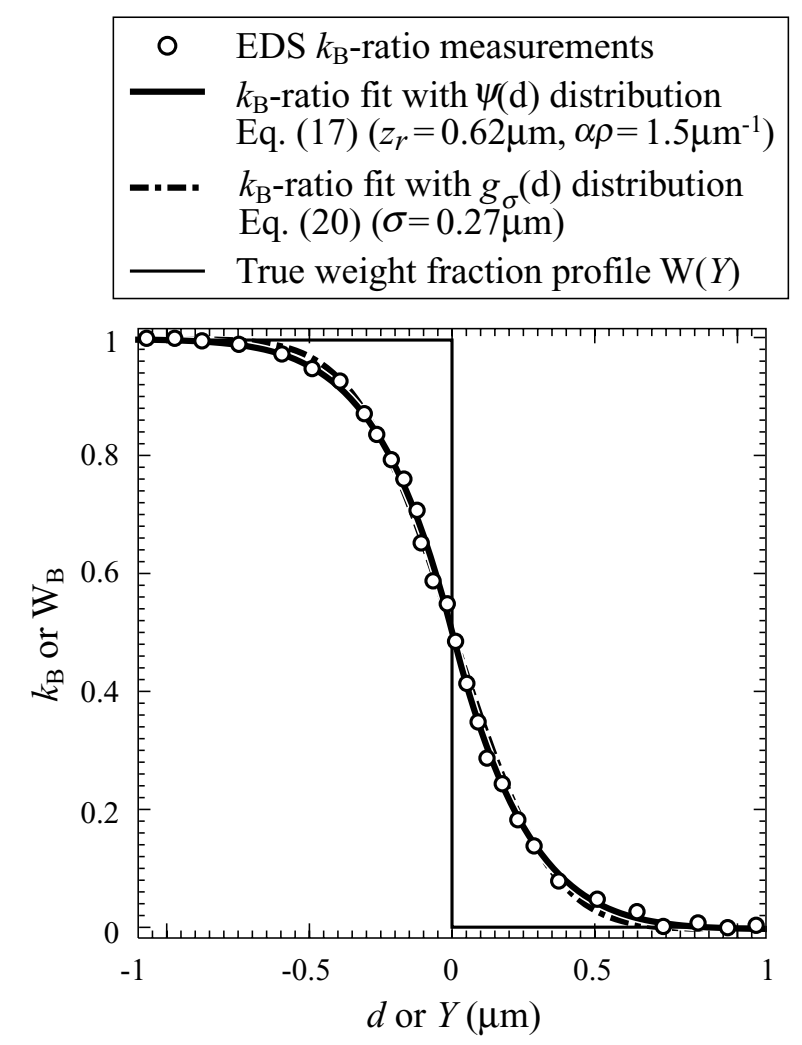

(a)
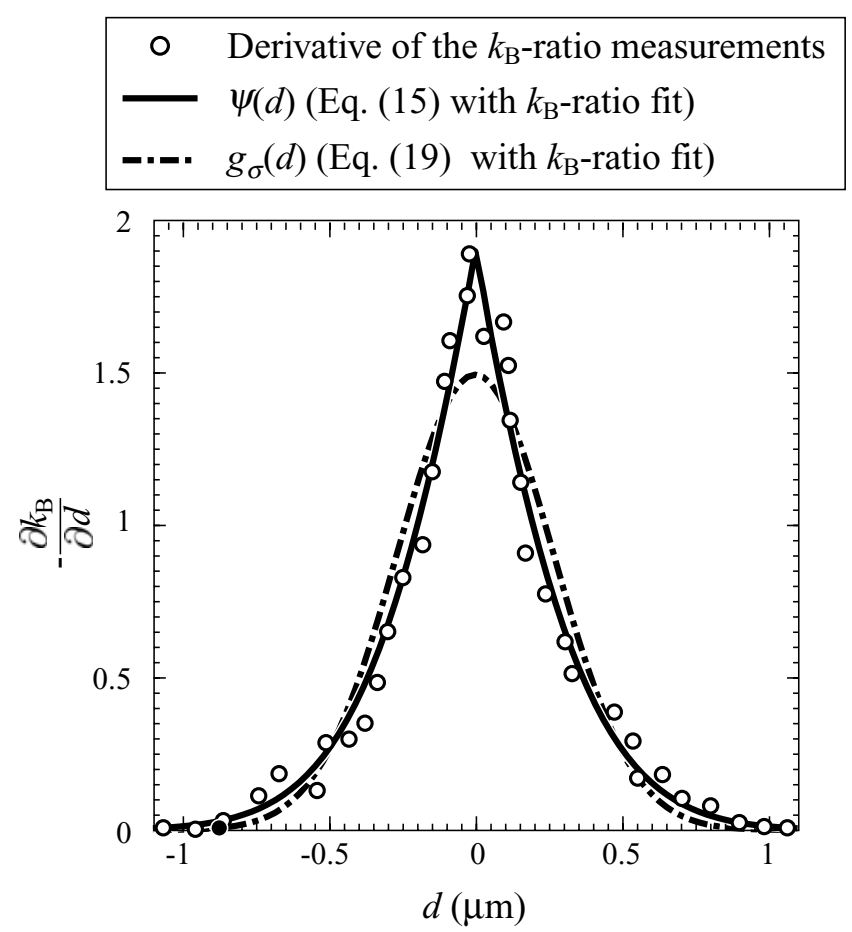

(b)

Figure 3: (a) Change of $k_{\mathrm{B}}$-ratio vs. $d$ for different lateral X-ray distributions fitted on EDS measurements when crossing a perfect interface of a $\mathrm{Ni}(\mathrm{A}) / \mathrm{Cu}(\mathrm{B})$ electrodeposited couple. Only a fraction of the measurement points is shown for the sake of clarity (total number: 44). (b) Corresponding lateral X-ray distributions. The derivative of the measured $k_{\mathrm{B}}$-ratio has been obtained by considering the average slope for 5 consecutive measurement points. $\left(\mathrm{E}_{0} \approx\right.$ $25 \mathrm{keV}, \delta \approx 8 \pi / 9, \theta \approx 29^{\circ}$ ) 


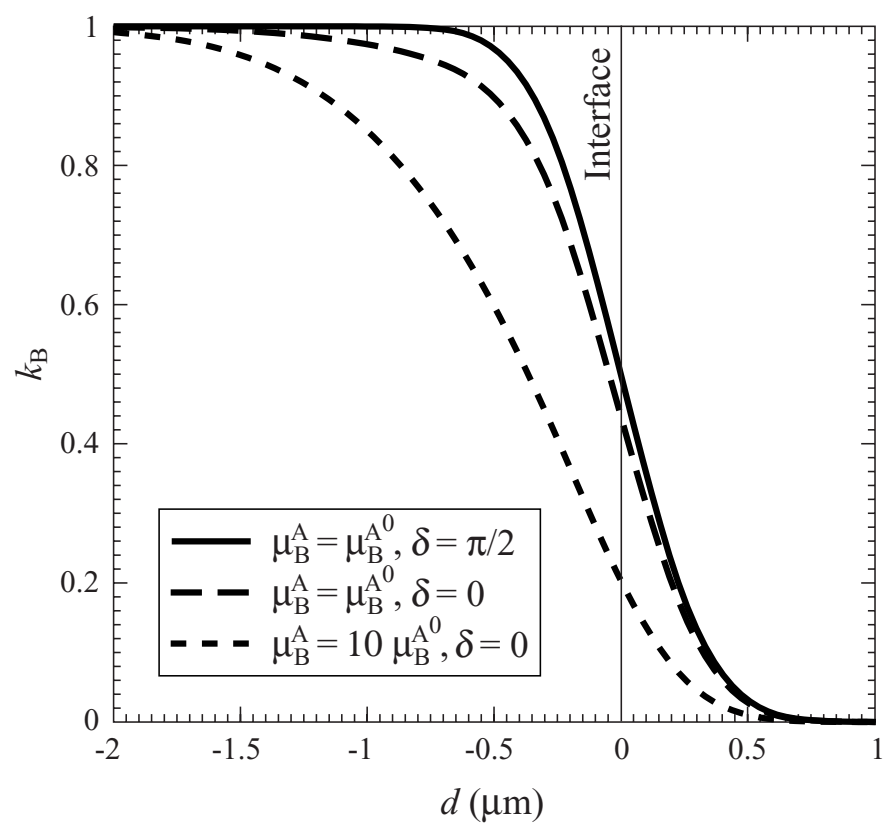

(a)

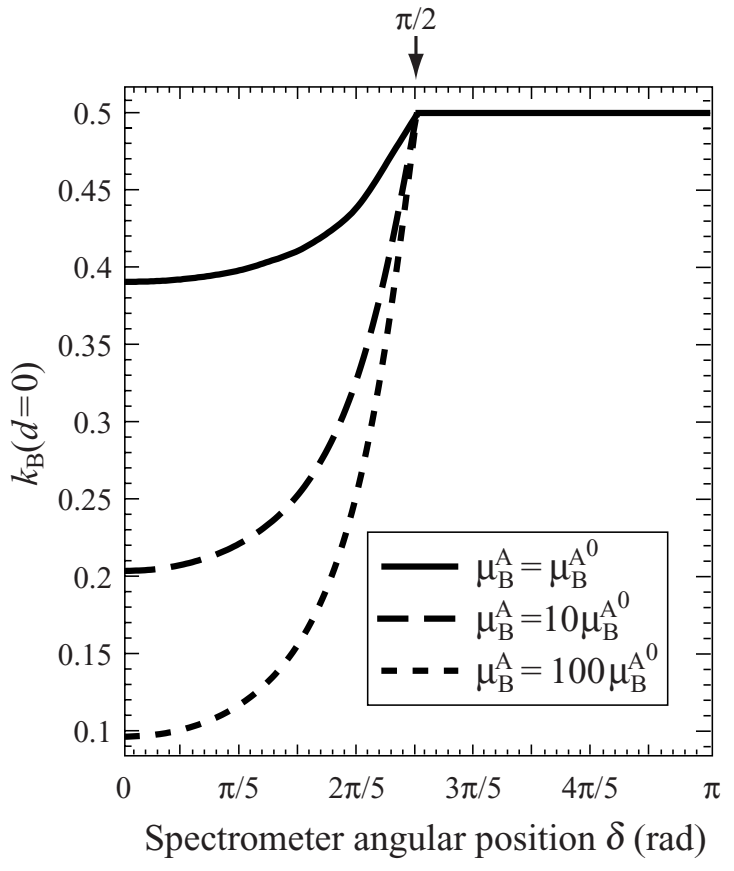

(b)

Figure 4: (a) Effect of the spectrometer angular position, $\delta$, and mass absorption coefficient, $\mu_{\mathrm{B}}^{\mathrm{A}}$, on the measured $k_{\mathrm{B}}(d)$-ratio (simulated with Eq. (21)) for a perfect interface. (b) Change of $k_{\mathrm{B}}$ for $d=0$ (i.e., primary electron beam focused on the interface) with spectrometer angle, $\delta$, for different absorption coefficients $\mu_{\mathrm{B}}^{\mathrm{A}}$ (Eq. (21)). All the other parameters correspond to a $\mathrm{Ni}(\mathrm{A}) / \mathrm{Cu}(\mathrm{B})$ couple for $\mathrm{E}_{0}=25 \mathrm{keV}, \theta=29^{\circ}$ and $\mu_{\mathrm{B}}^{\mathrm{A}^{0}}=\mu_{\mathrm{Cu}}^{\mathrm{Ni}}$. 


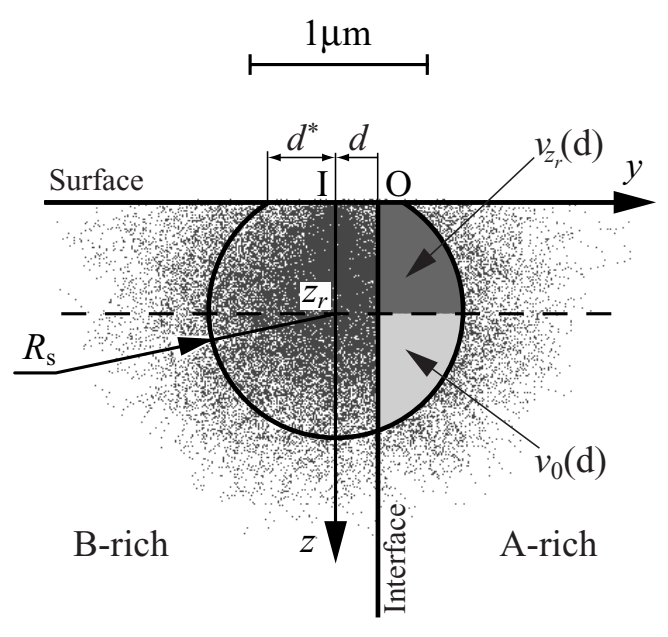

(a)

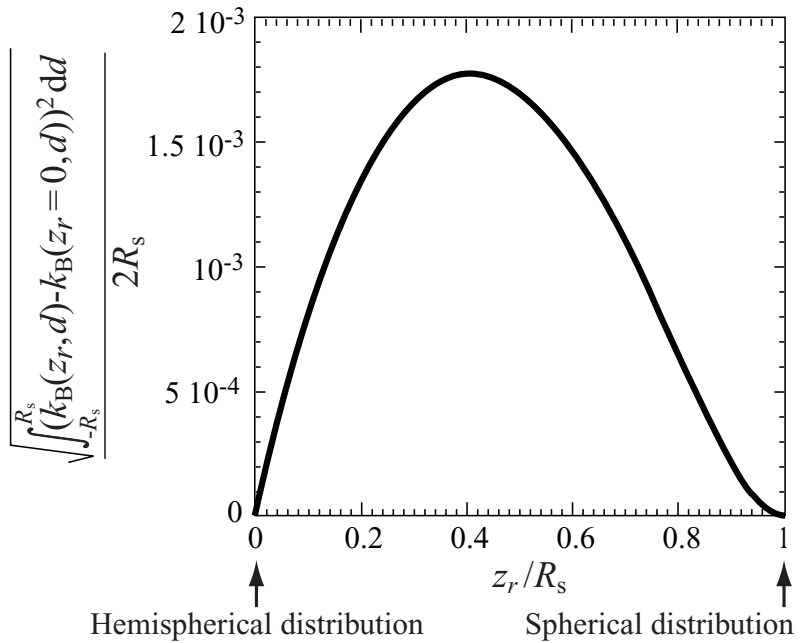

(b)

Figure 5: (a) Comparison between the Monte Carlo computation ${ }^{16}$ of the X-ray distribution for copper $\left(\mathrm{E}_{0}=25 \mathrm{keV}\right)$ with the approximated spherical one (sphere of radius $R_{\mathrm{s}}$ centered at a depth $z_{r}$ ). Gray surfaces define the partial volumes used in Eq. (23) when crossing a perfect interface. (b) Dimensionless root mean square error between a spherical X-ray emission distribution with a depth shift $z_{r}$ (Eq. (23)) and a hemispherical distribution, i.e., $z_{r}=0$ (Eq. (26)), vs. dimensionless depth shift $z_{r} / R_{\mathrm{S}}$. 


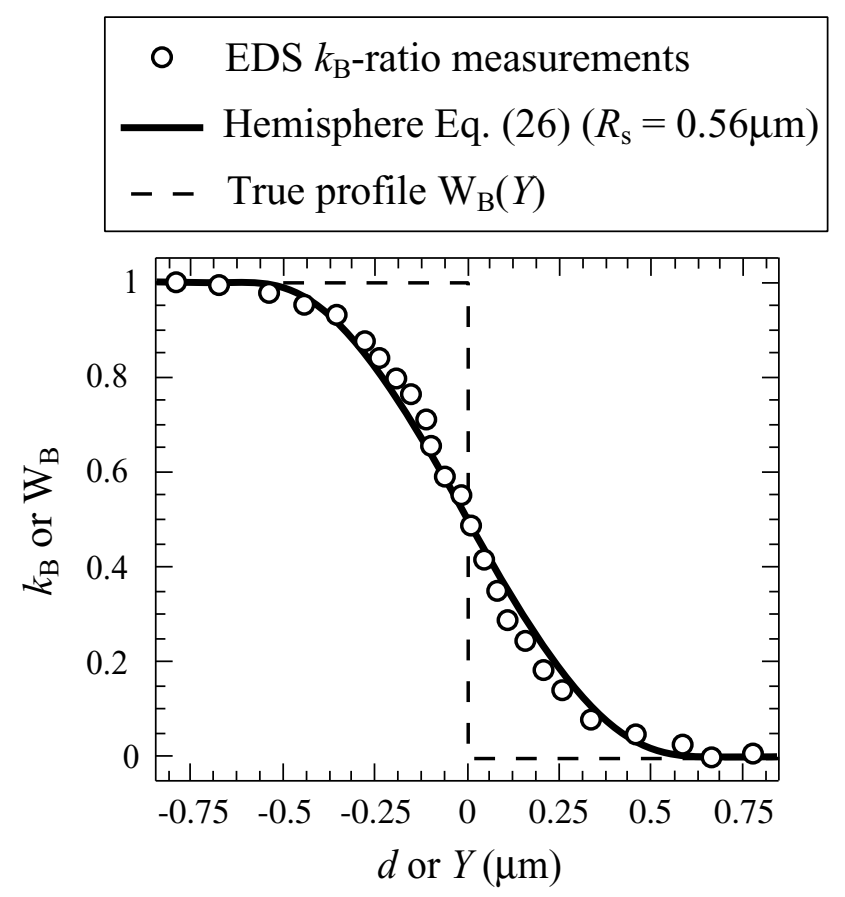

Figure 6: Determination of the radius $R_{\mathrm{S}}$ of the simplified spherical X-ray distribution by fitting the curve obtained with Eq. (26) on EDS measurements when crossing a perfect $\mathrm{Ni}(\mathrm{A}) / \mathrm{Cu}(\mathrm{B})$ interface $\left(\mathrm{E}_{0} \approx 25 \mathrm{keV}, \delta \approx 8 \pi / 9, \theta \approx 29^{\circ}\right)$. 


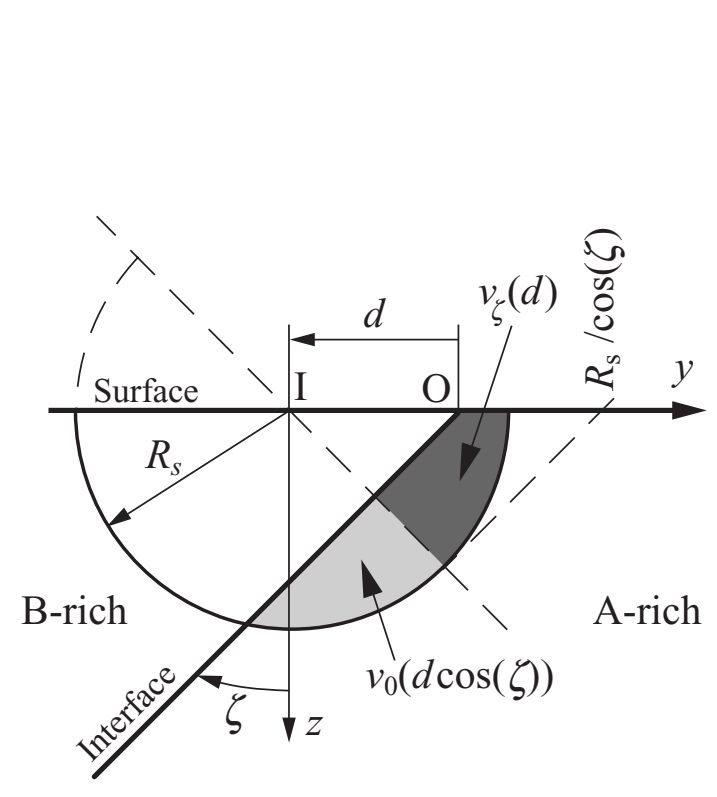

(a)

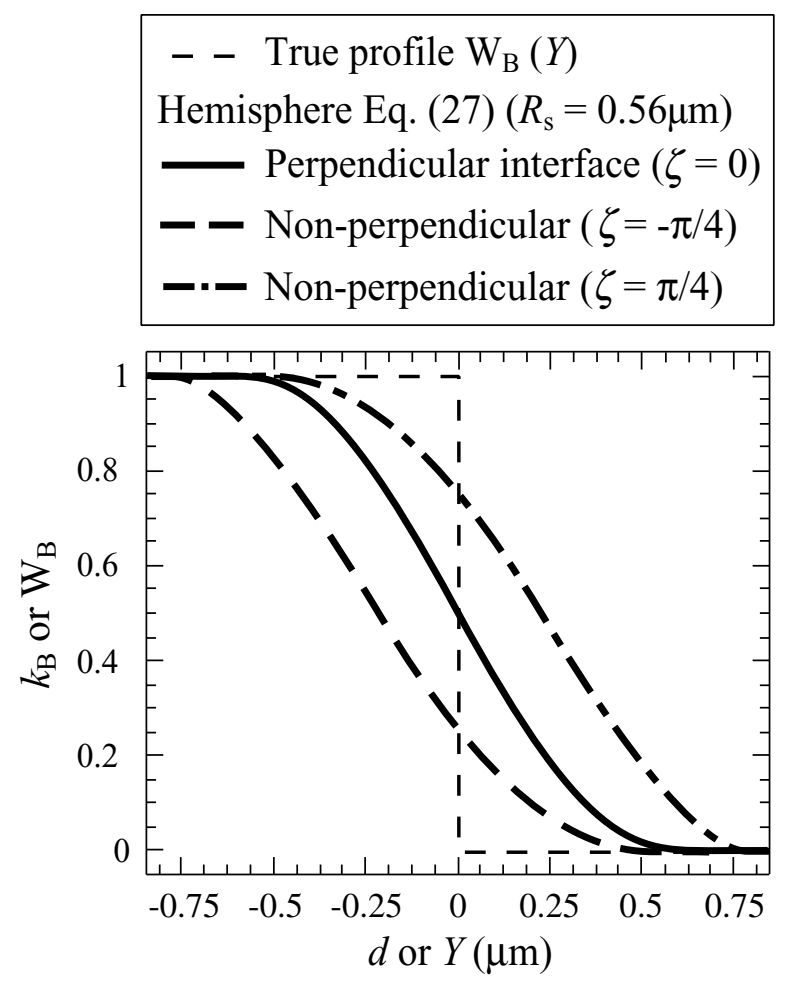

(b)

Figure 7: (a) Simplified hemispherical X-ray emission volume and the effect of a nonperpendicular interface ( $\zeta$ is positive and $d$ negative). Gray surfaces define the volume used in Eq. (27). (b) Simulated hemispherical effect for a perfect straight interface with a misorientation angle $\zeta$ obtained with Eq. (27). 


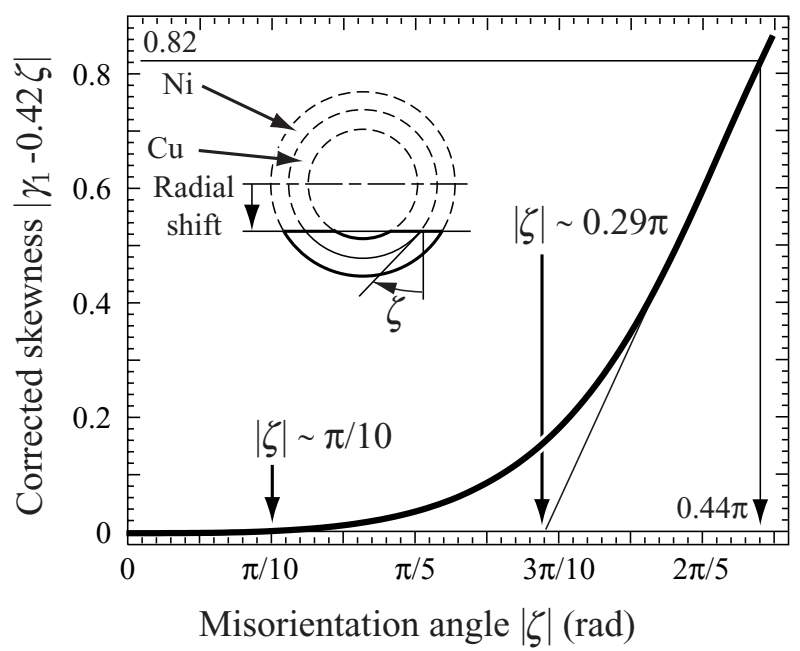

(a)
- EDS measurements

- Hemisphere Eq. (27) $\left(R_{\mathrm{s}}=0.56 \mu \mathrm{m}, \zeta=0.44 \pi\right)$

- - Estimated true profile $\mathrm{W}_{\mathrm{B}}(Y)$

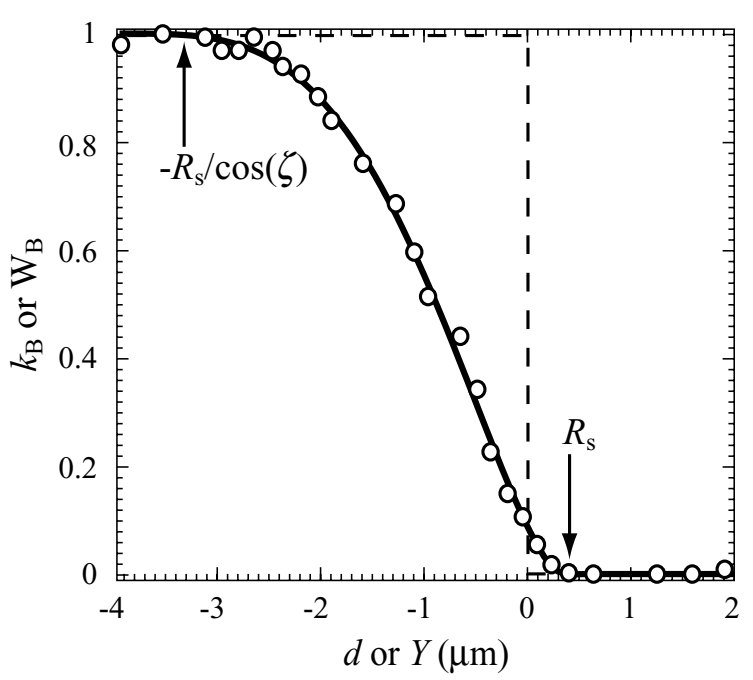

(b)

Figure 8: (a) Change in the corrected coefficient of asymmetry $\left|\gamma_{1}-0.42 \zeta\right|$ (Eq. (29)) with the misorientation angle $|\zeta|$ and schematic of the $\mathrm{Ni} / \mathrm{Cu}$ tubular specimen used to determine the misorientation angle $(\sim 0.44 \pi)$ from the corrected skewness $(0.82)$. (b) $k_{\mathrm{B}}$-ratio measurements on a $\mathrm{Ni}(\mathrm{A}) / \mathrm{Cu}(\mathrm{B})$ couple with a non-perpendicular interface $(\zeta \approx 0.4 \pi$ obtained by optical measurement) and the corresponding simulated $k_{\mathrm{B}}$-ratio with Eq. (27) and $\zeta \approx 0.44 \pi$ obtained by the measured skewness. Only a fraction of the measurement points is shown for the sake of clarity (total number: 54$)\left(\mathrm{E}_{0} \approx 25 \mathrm{keV}, \delta=\pi / 2, \theta \approx 29^{\circ}\right)$. 


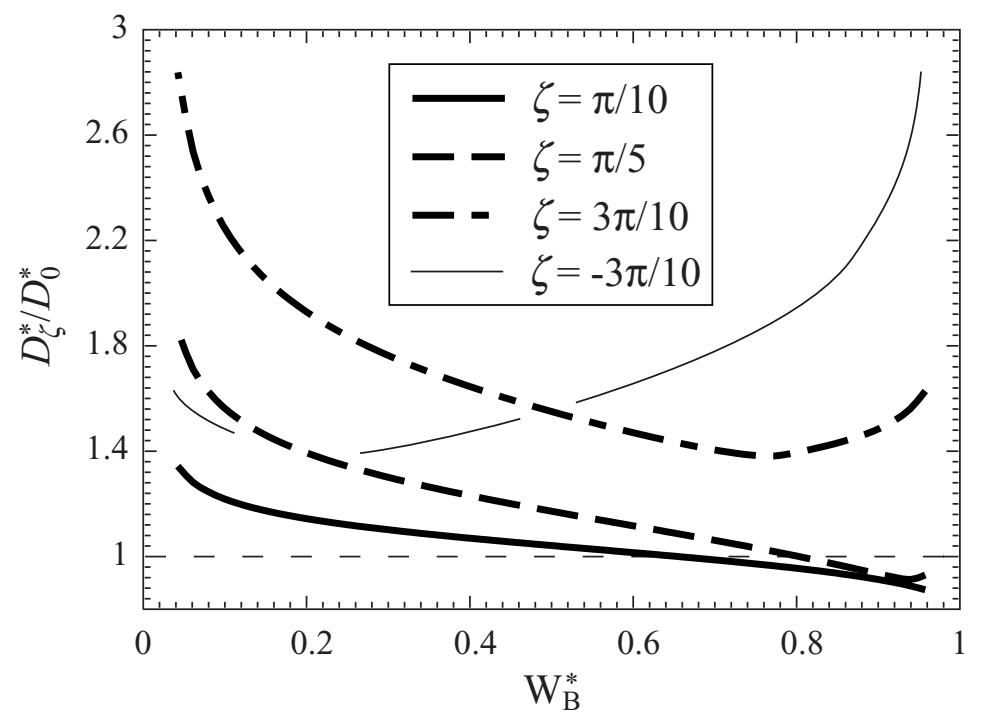

Figure 9: Apparent concentration dependence of the dimensionless diffusion coefficient due to a misoriented interface with no diffusion, $D_{\zeta}^{*} ; D_{0}^{*}$ stands for the apparent diffusion coefficient when measuring the concentration profile for a perfect interface with a hemispherical X-ray emission distribution. 


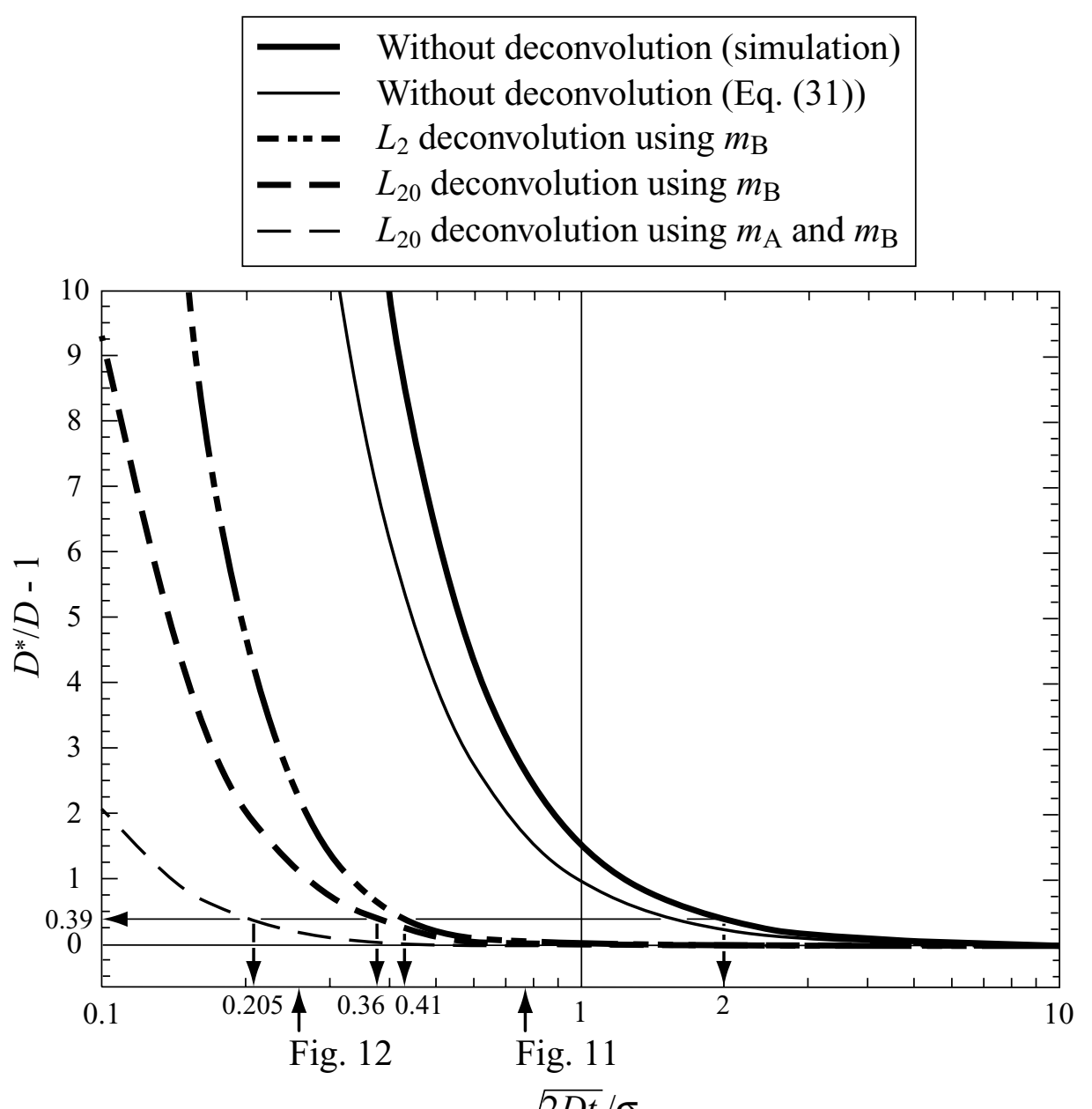

Figure 10: Error between the real diffusion coefficient, $D$, and the raw (without deconvolution) or deconvoluted ones, $D^{*}$, obtained by different deconvolution techniques vs. the ratio between the diffusion length, $\sqrt{2 D t}$, and the standard deviation of the lateral X-ray distribution $\sigma$ that evaluates the average effect. All the curves, except the second one, are obtained by simulating raw measurements with Eqs. (32) and (33) for a constant diffusion coefficient, $D$, curve (Eq. (49)) with a white noise of standard deviation $\sigma_{\mathrm{n}}=0.01$. The obtained measurement curves are discretized. The first curve is obtained without any deconvolution by assuming an apparent constant diffusion coefficient $D^{*}$ curve (Eq. (49)). The second is a direct plot of Eq. (31). The three following curves have been obtained by assuming an apparent constant diffusion coefficient $D^{*}$ curve (Eq. (49)) on deconvoluted weight fraction curves with different functionals and measurements, i.e., the first two curves use only the B-radiation measurements, $m_{\mathrm{B}}$, in the deconvolution functional whereas the last curve uses both the A and B-radiations measurements when the fluorescent A-radiations can be neglected (all material parameters are equivalent to a $\mathrm{Ni}(\mathrm{A}) / \mathrm{Cu}(\mathrm{B})$ couple with $\mathrm{E}_{0}=25 \mathrm{keV}, \sigma_{\mathrm{n}}=0.01, \delta=\pi / 2, \theta=40^{\circ}$ ). The regularization parameter $\lambda$ is obtained by the GCV method (Eq. (41)) and the second order derivative threshold $s$ for the $L_{20}$ deconvolution with Eq. (45). The downward arrows correspond to the resolution obtained without deconvolution and with the $L_{2}$ and $L_{20}$ ones. The $L_{2}$ deconvolution (with $m_{\mathrm{B}}$ only) allows one to improve the EPMA resolution by a factor 4.9 and the $L_{20}$ (with $m_{\mathrm{A}}$ and $m_{\mathrm{B}}$ ) one by a factor of 9.8 for the simplified cases used to plot this graph. 


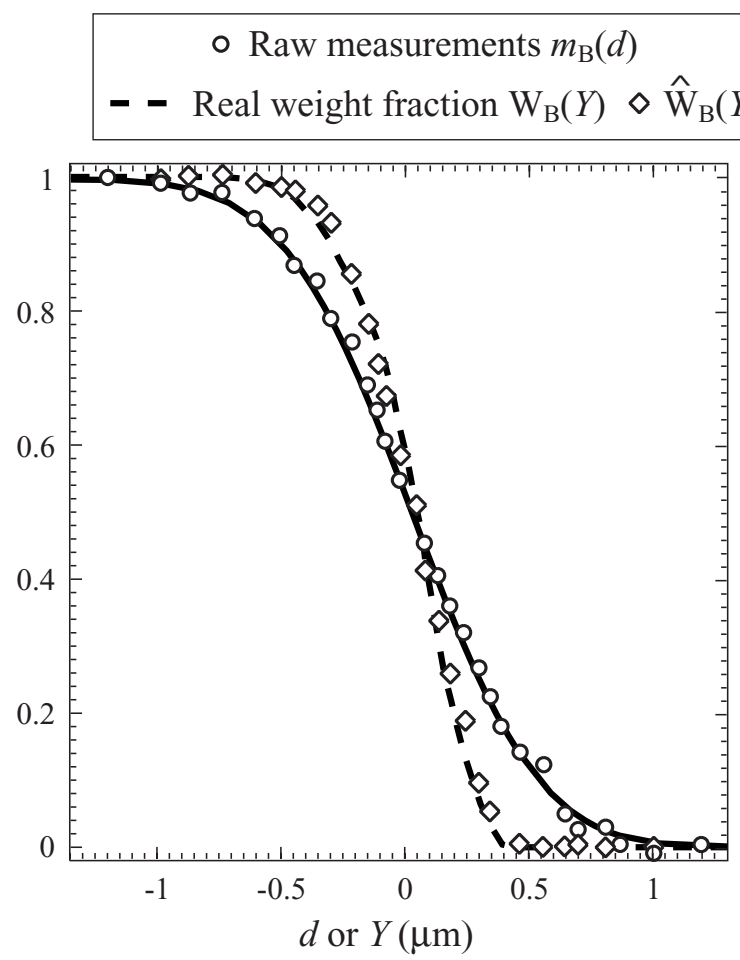

(a)
- Reconstructed measurements $k_{\mathrm{B}}(d)$

- $\hat{\mathrm{W}}_{\mathrm{B}}(Y)-L_{2}$ deconvolution

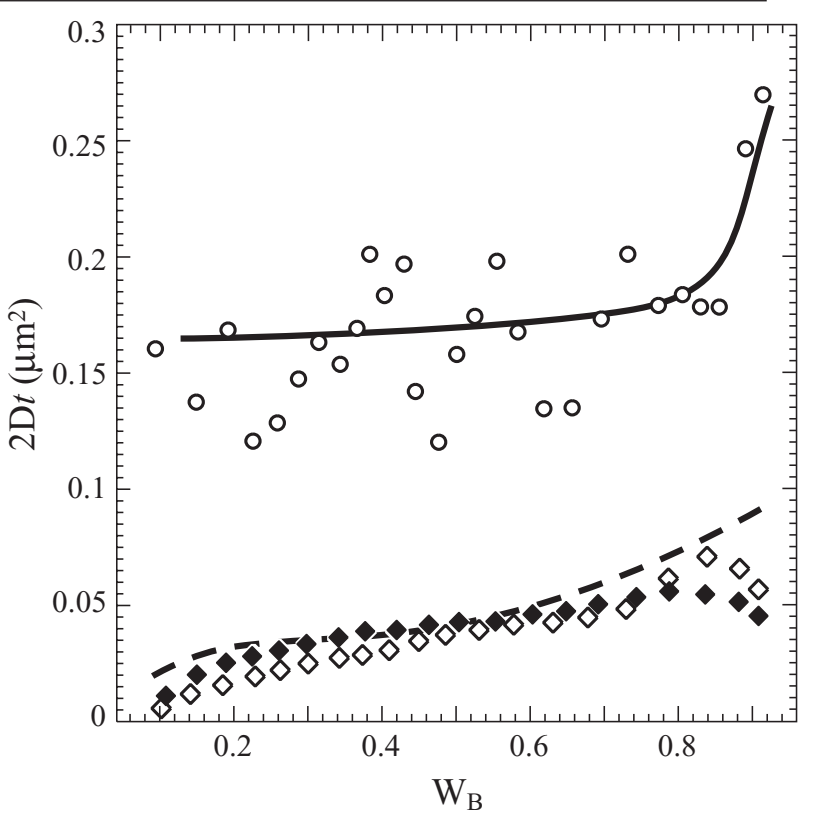

(b)

Figure 11: Regularized deconvolution of simulated raw B-radiations measurements (Eqs. (32) and (33)) for a real concentration-dependent diffusion coefficient of $\mathrm{Ni}(\mathrm{A}) / \mathrm{Cu}(\mathrm{B})$ electroplated diffusion couple ${ }^{27}$ at $550^{\circ} \mathrm{C}$ for 10 minutes $(\sqrt{2\langle D\rangle t} / \sigma \simeq 0.75$ ): (a) Weight fraction curves and deconvolution(s) when using the B-radiations measurements only. The two deconvolution techniques (Eqs. (38) and (43)) lead to very similar results (only the $L_{20}$ deconvolution is shown). The reconstructed measurements correspond to the $k$-ratio (Eq. (33)) obtained with the deconvoluted weight fraction profile obtained by $L_{20}$ deconvolution. Only a fraction of the points is shown for the sake of clarity (total number: 40). (b) Diffusion coefficient dependence with weight fraction in $\mathrm{Cu}(\mathrm{B})$ obtained by the Boltzmann-Matano method ${ }^{3}$ where the derivative is computed as the average slope of 6 consecutive points. The two deconvolution functionals yield the same acceptable behavior since the error between the real diffusion coefficients and the deconvoluted ones is less than the error arising from the measurement noise depicted by the scatter in the raw diffusion coefficient $\left(\sigma_{\mathrm{n}}=0.01, \mathrm{E}_{0}=25 \mathrm{keV}, \delta=\pi / 2, \theta=29^{\circ}, \lambda=\lambda_{\mathrm{GCV}} \approx 24\right.$, $s=s_{\text {opt }} \approx 49$ (Eq. (45))). 


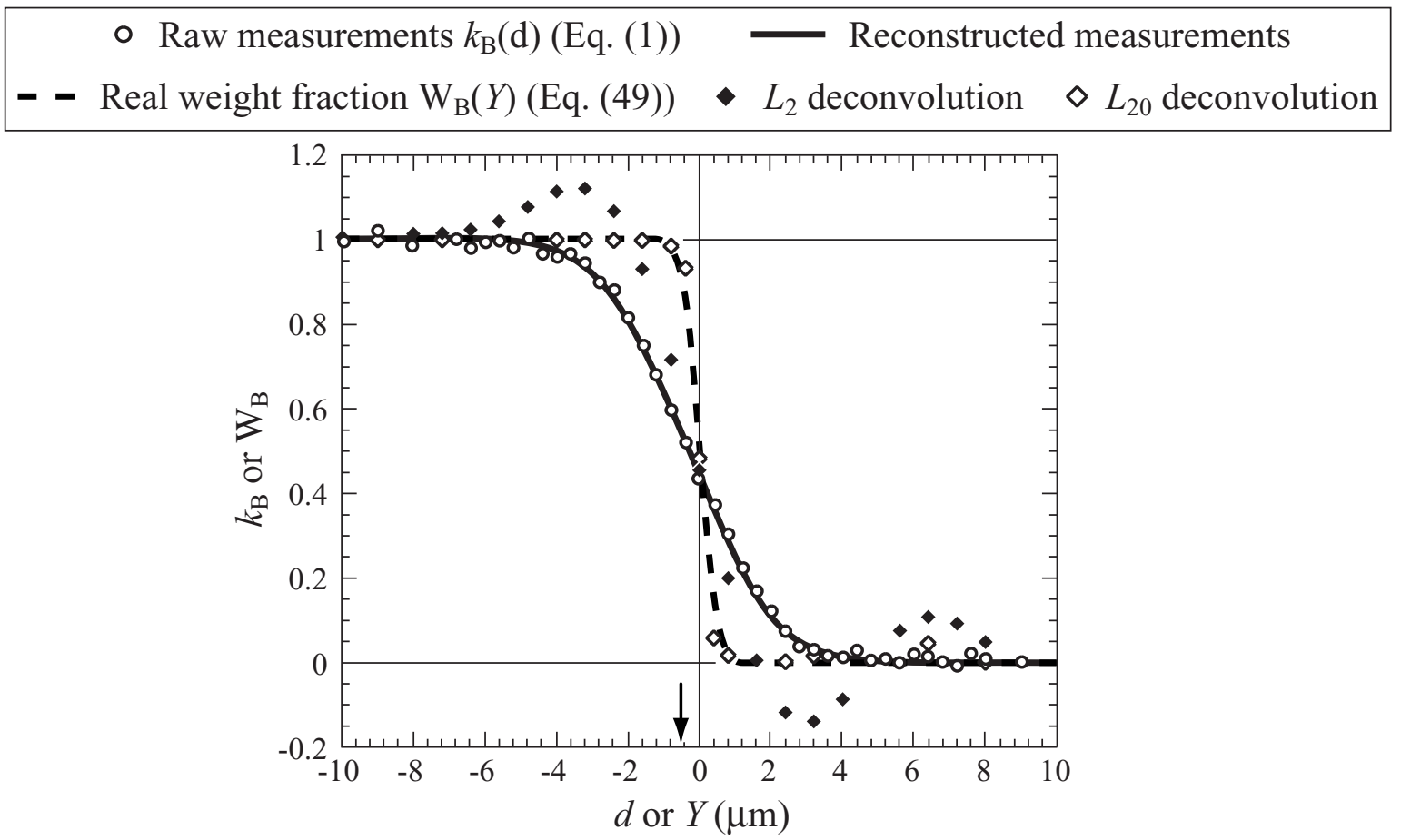

Figure 12: Regularized deconvolution of simulated raw measurements (Eqs. (32) and (33)) for a weight fraction curve with a constant diffusion coefficient (Eq. (49)) when $\sqrt{2 D t} / \sigma=0.24$. Comparison between the $L_{2}$ deconvolution when using the B-radiations measurements only (Eqs. (38)) and $L_{20}$ one when using both A and B-radiations measurements (Eqs. (34) and (43)). The reconstructed measurements correspond to the $k$-ratio obtained with the deconvoluted weight fraction profile obtained by $L_{20}$ deconvolution (Eq. (33)). The downward arrow corresponds to the position of the Matano interface ${ }^{3}$ for the raw measurement curve whereas its real position (determine with the real weight fraction curve) is located at $Y=0$ $\left(\sigma_{\mathrm{n}}=0.01, \mathrm{E}_{0}=25 \mathrm{keV}, \delta=\pi / 2, \theta=29^{\circ}\right.$, material parameters correspond to a $\mathrm{Ni}(\mathrm{A}) / \mathrm{Cu}(\mathrm{B})$ couple except $\mu_{\mathrm{B}}^{\mathrm{A}}=15 \mu_{\mathrm{Cu}}^{\mathrm{Ni}}$ to increase the absorption effect, $\lambda=\lambda_{\mathrm{GCV}} \approx 47, s=s_{\text {opt }} \approx 0.2$ (Eq. (45))). Only a fraction of the points (total number: 45) and the B-curves are shown for the sake of clarity . 


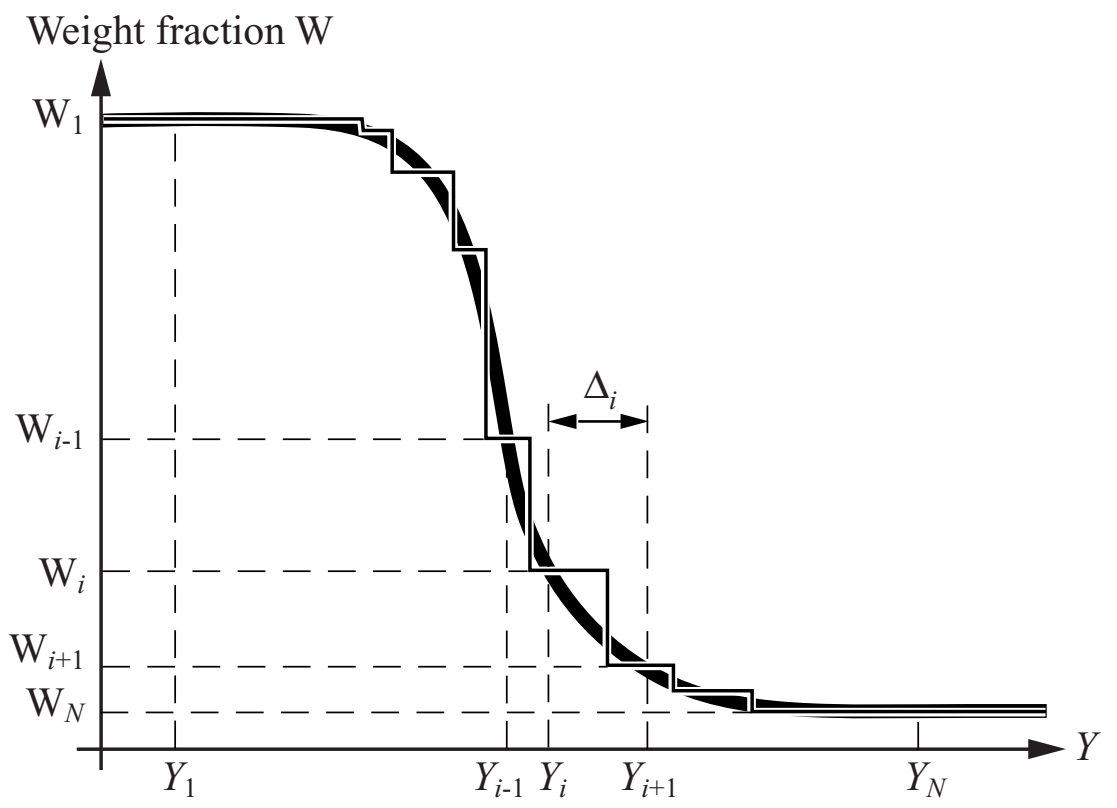

Figure 13: Typical concentration profile and its spatial discretization with stepwise constant functions corresponding to measurement points. 


\section{List of Tables}

1 Example of parameters used for the simulated annealing algorithm determined with the deconvolution problem of Fig. 11. See Goffe et al. ${ }^{44}$ for description and practical method for finding the best parameters. . . . . . . . . . .
Sensitivity analysis for the $L_{2}$ and $L_{20}$ deconvolution methods (when using

2 Sensitivity analysis for the $L_{2}$ and $L_{20}$ deconvolution methods (when using $m_{\mathrm{B}}$ only) to the smoothing parameter $\lambda$ and the threshold for the second order derivative $s$ with the deconvolution problem of Fig. 11. $\lambda_{\mathrm{GCV}}$ stands for the value obtain with the Generalized Cross Validation method ${ }^{33,31}$ (Eq. (41)), $s_{\text {opt }}=2 D^{*} t /\left(\sigma^{2} \min _{i} \Delta_{i}\right)$ (Eq. (45)) and $\mathscr{E}^{2}(\lambda, s)$ is the mean square error between the real weight fraction curve and the deconvoluted one for any couple $(\lambda, s)\left(\right.$ Eq. (46)) with $\mathscr{E}_{\text {ref }}^{2}=\mathscr{E}^{2}\left(\lambda_{\mathrm{GCV}}, s_{\text {opt }}\right) \ldots \ldots \ldots \ldots . \ldots . \ldots . . \ldots 44$

3 Sensitivity analysis for the $L_{20}$ deconvolution method (when using $m_{\mathrm{A}}$ and $m_{\mathrm{B}}$ ) to the smoothing parameter $\lambda$ and the threshold for the second order derivative $s$ with the deconvolution problem of Fig. 12. $\lambda_{\mathrm{GCV}}$ stands for the value obtain with the Generalized Cross Validation method ${ }^{33,31}$ (Eq. (41)), $s_{\text {opt }}=2 D^{*} t /\left(\sigma^{2} \min _{i} \Delta_{i}\right)($ Eq. $(45))$ and $\mathscr{E}^{2}(\lambda, s)$ is the mean square error between the real weight fraction curve and the deconvoluted one for any couple $(\lambda, s)$ (Eq. (46)) with $\mathscr{E}_{\text {ref }}^{2}=\mathscr{E}^{2}\left(\lambda_{\mathrm{GCV}}, s_{\text {opt }}\right) \ldots \ldots \ldots \ldots$ 
Table 1: Example of parameters used for the simulated annealing algorithm determined with the deconvolution problem of Fig. 11. See Goffe et al. ${ }^{44}$ for description and practical method for finding the best parameters.

\begin{tabular}{c|l|c} 
Parameter & Description & Value \\
\hline$\Delta \varepsilon$ & Convergence criterion & $10^{-4}$ \\
$T_{0}$ & Initial temperature & 10 \\
$r_{T}$ & Temperature reduction factor & 0.85 \\
$N_{T}$ & Time through $N_{S}$ loops before temperature reduction & 10 \\
$N_{S}$ & Time through function before length adjustment of $V_{i}$ & 10 \\
$N_{\varepsilon}$ & Time function tolerance is achieved before termination & 4 \\
$C_{i}$ & Length adjustment factor (controls how fast $V_{i}$ is adjusted) & $2(i=1, N)$ \\
$V_{i}$ & Step length for the weight fraction & $0.5(i=1, N)$ \\
\hline
\end{tabular}


Table 2: Sensitivity analysis for the $L_{2}$ and $L_{20}$ deconvolution methods (when using $m_{\mathrm{B}}$ only) to the smoothing parameter $\lambda$ and the threshold for the second order derivative $s$ with the deconvolution problem of Fig. 11. $\lambda_{\mathrm{GCV}}$ stands for the value obtain with the Generalized Cross Validation method ${ }^{33,31}$ (Eq. (41)), $s_{\text {opt }}=2 D^{*} t /\left(\sigma^{2} \min _{i} \Delta_{i}\right)$ (Eq. (45)) and $\mathscr{E}^{2}(\lambda, s)$ is the mean square error between the real weight fraction curve and the deconvoluted one for any couple $(\lambda, s)\left(\right.$ Eq. (46)) with $\mathscr{E}_{\text {ref }}^{2}=\mathscr{E}^{2}\left(\lambda_{\mathrm{GCV}}, s_{\text {opt }}\right)$.

\begin{tabular}{|cc||c|c|c|c|c|c|c|c|c|}
\hline \multicolumn{2}{|c||}{$\lambda / \lambda_{\mathrm{GCV}}$} & $1 / 16$ & $1 / 8$ & $1 / 4$ & $1 / 2$ & 1 & 2 & 4 & 8 & 16 \\
\hline $\mathscr{E}^{2}\left(\lambda, s_{\text {opt }} / \mathscr{E}_{\text {ref }}^{2}\right.$ & $L_{20}$ & 1.6 & 1.38 & 1.2 & 1.075 & 1 & 0.974 & 1.04 & 1.16 & 1.63 \\
\hline $\mathscr{E}^{2}(\lambda) / \mathscr{E}_{\text {ref }}^{2}$ & $L_{2}$ & 1.44 & 1.24 & 1.1 & 1.02 & 1 & 1.04 & 1.13 & 1.31 & 1.63 \\
\hline \hline \multicolumn{2}{|c||}{$s_{\text {opt }}$} & $1 / 16$ & $1 / 8$ & $1 / 4$ & $1 / 2$ & 1 & 2 & 4 & 8 & 16 \\
\hline $\mathscr{E}^{2}\left(\lambda_{\mathrm{GCV}}, s\right) / \mathscr{E}_{\text {ref }}^{2}$ & $L_{20}$ & 1.023 & 1.015 & 1.011 & 1.005 & 1 & 0.99 & 0.99 & 0.99 & 0.99 \\
\hline
\end{tabular}

Table 3: Sensitivity analysis for the $L_{20}$ deconvolution method (when using $m_{\mathrm{A}}$ and $m_{\mathrm{B}}$ ) to the smoothing parameter $\lambda$ and the threshold for the second order derivative $s$ with the deconvolution problem of Fig. 12. $\lambda_{\mathrm{GCV}}$ stands for the value obtain with the Generalized Cross Validation method ${ }^{33,31}$ (Eq. (41)), $s_{\text {opt }}=2 D^{*} t /\left(\sigma^{2} \min _{i} \Delta_{i}\right)$ (Eq. (45)) and $\mathscr{E}^{2}(\lambda, s)$ is the mean square error between the real weight fraction curve and the deconvoluted one for any couple $(\lambda, s)\left(\right.$ Eq. (46)) with $\mathscr{E}_{\text {ref }}^{2}=\mathscr{E}^{2}\left(\lambda_{\mathrm{GCV}}, s_{\text {opt }}\right)$.

\begin{tabular}{|cc||c|c|c|c|c|c|c|c|c|}
\hline$\lambda / \lambda_{\mathrm{GCV}}$ & $1 / 100$ & $1 / 8$ & $1 / 4$ & $1 / 2$ & 1 & 2 & 4 & 8 & 100 \\
\hline $\mathscr{E}^{2}\left(\lambda, s_{\mathrm{opt}}\right) / \mathscr{E}_{\mathrm{ref}}^{2}$ & $L_{20}$ & 3.8 & 0.9 & 0.92 & 0.96 & 1 & 1.02 & 1.04 & 1.05 & 21.4 \\
\hline \hline$s / s_{\text {opt }}$ & $1 / 16$ & $1 / 8$ & $1 / 4$ & $1 / 2$ & 1 & 2 & 4 & 8 & 16 \\
\hline $\mathscr{E}^{2}\left(\lambda_{\mathrm{GCV}}, s\right) / \mathscr{E}_{\mathrm{ref}}^{2}$ & $L_{20}$ & 3.6 & 3.34 & 1.17 & 1.16 & 1 & 1.64 & 1.56 & 1.59 & 1.6 \\
\hline
\end{tabular}

